Tserkovniuk R.G., Gozhenko A.I., Korolyshyn T.A., Kovbasnyuk M.M., Hubyts'kyi V.Y., Kikhtan V.V., Fil V.M., Anchev A.S., Zukow W., Yanchij R.I., Popovych I.L. Relationships between geomagnetic Ap-index and parameters of the acupuncture points as well as neuroendocrine-immune complex in patients with its dysfunction. Journal of Education, Health and Sport. 2021;11(12):405432. eISSN 2391-8306. DOI http://dx.doi.org/10.12775/JEHS.2021.11.12.034

https://apcz.umk.pl/czasopisma/index.php/JEHS/article/view/JEHS.2021.11.12.034

https://zenodo.0rg/record/5859929

\title{
Relationships between geomagnetic Ap-index and parameters of the acupuncture points as well as neuroendocrine-immune complex in patients with its dysfunction
}

\author{
R.G. Tserkovniuk ${ }^{1}$, A.I. Gozhenko ${ }^{1}$, T.A. Korolyshyn ${ }^{1,2,3}$, M.M. Kovbasnyuk ${ }^{2}$, \\ V.Y. Hubyts'kyi ${ }^{3}$, V.V. Kikhtan ${ }^{3}$, V.M. Fil ${ }^{4}$, A.S. Anchev ${ }^{1}$, W. Zukow ${ }^{5}$, R.I. Yanchij ${ }^{2}$, \\ I.L. Popovych ${ }^{1,2}$ \\ ${ }^{1}$ Ukrainian Research Institute of Medicine for Transport, Odesa, Ukraine \\ ruslan.urology@gmail.com prof.gozhenko@gmail.com \\ ${ }^{2}$ OO Bohomolets' Institute of Physiology, Kyïv, Ukraine \\ i.popovych@biph.kiev.ua janchii2014@gmail.com \\ ${ }^{3}$ Clinical sanatorium "Moldova", Truskavets', Ukraine \\ ${ }^{4}$ Ivan Franko Pedagogical University, Drohobych, Ukraine fillvitalij@gmail.com \\ ${ }^{5}$ Nicolaus Copernicus University, Torun, Poland w.zukow@wp.pl
}

\begin{abstract}
Background. Back in 1990, YuP Limansky hypothesized acupuncture points (AP) as polymodal receptors of the ecoceptive sensitivity system. In the process of hypothesis development in 2003 an existence of separate functional system of regulation of electromagnetic balance of organism has been substantiated and a working conception of light therapy has been formulated. In line with this hypothesis, we set out to analyze the relationships between the disturbances of the geomagnetic field (Apindex) and the electrical conductivity of a number of AP, on the one hand, and the parameters of the neuroendocrine-immune complex in patients with its dysfunction, on the other. Material and methods. The object of observation were 21 men (24-63 y) and 20 women (30-72 y) with neuroendocrine-immune complex dysfunction. Each patient was tested twice with an interval of 4 days. Retrospectively we recorded the geomagnetic Ap-Index on the day of testing and during the previous 7 days, using resource https://www.spaceweatherlive.com/. Recorded the electrical conductivity of 9 pairs of AP, electroencephalogram (EEG) and heart rate variability (HRV) parameters, determined the plasma level of cortisol, triiodothyronine and testosterone, the content of lymphocytes expressing CD3, CD4, CD25, CD8, CD22 and CD56 receptors, the serum level of circulating immune complexes, immunoglobulins classes M, G, A as well as C-reactive protein, IL-1 $\beta$ and IL-6. The state of phagocytic function of neutrophils estimated by microbial count and phagocytic and killing indices against Staphylococcus aureus and Escherichia coli. Results. During the week, the average level of Ap-index ranged from 7 to $13 \mathrm{nT}$. Maximum coefficients of multiple correlation with APs parameters were detected for Ap-index on 6 day before $(\mathrm{R}=0,552)$ and on the day of testing $(R=0,470)$, with EEG parameters on the eve of registration $(R=0,708)$ and on 6 day before its $(\mathrm{R}=0,685)$, with immunity parameters on the eve of blood sampling $(\mathrm{R}=0,768)$ and on 5 day before its $(\mathrm{R}=0,758)$, with HRV\&Hormonal parameters on $2(\mathrm{R}=0,506)$ and $7(\mathrm{R}=0,403)$ days before testing. The canonical correlation between Apindices for 7 days before and on the day of testing, and the parameters APs is 0,661; EEG parameters is 0,886; HRV\&Hormonal parameters is 0,766 and immunity parameters is 0,921 . APs parameter are closely related to the EEG $(\mathrm{R}=0,997)$ and HRV\&Hormonal parameters $(\mathrm{R}=0,740)$. In turn, the immune parameters are closely related to the EEG $(\mathrm{R}=0,944)$ and HRV\&Hormonal parameters $(\mathrm{R}=0,714)$. Conclusion. Disturbances of the geomagnetic field (Ap-index) causes a significant immediate modulating effect on the parameters of neuroendocrine-immune complex, apparently through acupuncture points as polymodal receptors of the ecoceptive sensitivity system.
\end{abstract}

Key words: geomagnetic Ap-index, acupuncture points, neuroendocrine-immune complex, relationships, humans. 


\title{
INTRODUCTION
}

\author{
We dedicate this article to two geniuses \\ who are not recognized for life and even \\ persecuted, but without whom this study \\ would never have occurred to us.
}

Alexandr L. Chizhevsky, the founder of Heliobiology.

Yuriy P. Limansky, the author of hypothesis about the AP as polymodal receptors of the ecoceptive sensitivity system.

In 1936, the founder of the Heliobiology Chizhevsky suggested correlation of some biological processes on the Earth with cycles of Solar activity [1]. Unfortunately, newborn science suffered the same fate as Soviet genetics ...

The geomagnetic field is a fundamental nature of the planet that is produced by the geodynamo of the Earth's outer core. There is evidence that changes in this field can affect biological systems [2,3]. While geomagnetic field deflects the solar wind particles, any changes in the density or the velocity of the solar wind interact with the magnetosphere and cause temporary alterations in the field that is measurable at the Earth surface [4]. This phenomenon is called geomagnetic disturbance or geomagnetic activity. The concept of space weather is relatively new and the main researches about this phenomenon have been done in recent four decades. Space weather defined as the conditions in space that affect Earth, consequences of flowing ionized particle of the solar wind against geomagnetic field. However, geomagnetic field acts like a shield and deflect most of the solar charged particles, it is also impressed and altered by solar wind. These geomagnetic field alterations are called geomagnetic disturbances. For quantifying geomagnetic disturbances, several indices such as Planetary K index $(\mathrm{Kp})$ and Planetary A index (Ap) were defined [5].

Back in 1990, Limansky [6] hypothesized acupuncture points (AP) as polymodal receptors of the ecoceptive sensitivity system. In the process of hypothesis development in 2003 an existence of separate functional system of regulation of electromagnetic balance of organism has been substantiated and a working conception of light therapy has been formulated [7]. As a basis, there is a possibility to use the AP for input of biologically necessary electromagnetic waves into the system of their conductors in a body that might be considered as a transport facility for energy of the polarized electromagnetic waves. Zones-recipients are organ shaving an electromagnetic dysbalance due to excess of biologically inadequate radiation and being the targets for peroxide oxidation. Foremost, a body has the neuro-hormonal and immune regulatory systems. Electromagnetic stimulation or modification of functions of the zones-recipients determines the achievement of therapeutic and useful effects, and their combination with local reparative processes allows to attain a clinical goal. Authors show the experimental facts in support of a hypothesis that a living organism can perceive an action of the electromagnetic fields of optical range not only via the visual system, but also through the off-nerve receptors (specific energy-sensitive proteins detecting critical changes of energy in cells and functioning as the "sensory" cell systems), as well as via the AP.

Recently, on the example of patients with multiple sclerosis and radiculopathy, we found an immediate immunotropic effects of the disturbances of the geomagnetic field by analyzing the relationships between immunity parameters and the Ap-index. The canonical correlation between Ap-indices for 7 days before and on the day of blood collection, on the one hand, and the levels of $\mathrm{CD} 4^{+}, \mathrm{CD} 56^{+}, \mathrm{CD} 25^{+}$and $\mathrm{CD} 8^{+}$lymphocytes and the concentration of IgM and IL- $\beta$ - on the other hand, was very strong $(\mathrm{R}=0,741)$. Thus, disturbances of the geomagnetic field causes a significant immediate modulating effect on the level of immune parameters in the blood, mostly T-helpers (suppressing) and natural killers (enhancing) [8].

The data available in the literature give grounds for assumptions about the direct effect of disturbances of the geomagnetic field on immunocytes, and indirectly, through immunotropic neurotransmitters and hormones.

Our hypothesis is as follows. Disturbances of the geomagnetic field are perceived by AP. The information obtained is transmitted to neurons and endocrinocytes, the mediators of which, in turn, affect immunocytes. The purpose of this study is to test this hypothesis.

\section{MATERIAL AND METHODS}

The object of observation were 21 men $(24-63 ; 44 \pm 12$ y) and 20 women $(30-72 ; 49 \pm 12$ y) with neuroendocrine-immune complex dysfunction: increased level of HRVs-markers of sympathetic tone and decreased markers of vagal tone, moderate hypocortisolemia in both sexes and hypertestosteronemia in women (see Table 10), decreased parameters of phagocytosis by neutrophils of gram-negative and gram-positive bacteria as well as the level of T-helpers, but increased levels of NK- and B-lymphocytes, Igg G and M (see Table 12). Each patient was examined twice with an interval of 4 days. Observations were carried out on 09.06. and 13.06. 2015 (13 men and 3 women), 14.09. and 18.09. 2015 (1 man and 4 women), 27-28.03. and 04-05.04. 
2018 ( 3 men and 7 women), 28.01 and 01.02. 2019 (4 men and 6 women). Retrospectively we recorded the geomagnetic Ap-Index, i.e. the average value of deviation of the geomagnetic field strength from normal in this region (range on Earth: 35-65 $\mu \mathrm{T}$ ) on the day of testing and during the previous 7 days, using a publicly available information resource https://www.spaceweatherlive.com/.

Electrical conductivity recorded (VY Hubyts'kyi) in follow acupuncture points: $\operatorname{Pg}(\mathrm{ND}), \mathrm{TR}(\mathrm{X})$, MC(AVL), C(V), LY, Dg(Al), GI, IG, SPE at Right and Left side. Used complex "Medissa". For each pair, the Laterality Index (LI) was calculated according to the formula:

LI, $\%=[200 \bullet($ Right - Left $) /($ Right + Left $)]$.

To assess the parameters of heart rate variability (HRV) recorded (TA Korolyshyn) during 7 min electrocardiogram in II lead (software and hardware complex "CardioLab+HRV", KhAI-MEDICA, Kharkiv). For further analysis the following parameters HRV were selected. Temporal parameters (Time Domain Methods): the standard deviation of all $\mathrm{NN}$ intervals (SDNN), the square root of the mean of the sum of the squares of differences between adjacent NN intervals (RMSSD), the percent of interval differences of successive NN intervals greater than $50 \mathrm{msec}\left(\mathrm{pNN}_{50}\right)$; Triangular Index (TNN). Spectral parameters (Frequency Domain Methods): power spectral density (PSD) bands of HRV - high-frequency (HF, range 0,4 $\div 0,15 \mathrm{~Hz}$ ), lowfrequency (LF, range $0,15 \div 0,04 \mathrm{~Hz}$ ), very low-frequency (VLF, range $0,04 \div 0,015 \mathrm{~Hz}$ ) and ultralow-frequency (ULF, range $0,015 \div 0,003 \mathrm{~Hz}$ ). Calculated classical indexes: LF/HF, LFnu=100\%・LF/(LF+HF) $[9,10]$. Baevsky's parameters. Heart rate (HR), Mode (Mo), the Amplitude of Mode (AMo), Variational Sweep (MxDMn), Vegetative Balance Index (VBI=AMo/MxDMn), Stress Index (BSI=AMo/2•Mo•MxDMn) as well as Baevsky’s Activity of Regulatory Systems Index (BARS) [11].

Simultaneosly EEG recorded (TA Korolyshyn) a hardware-software complex "NeuroCom Standard" (KhAI MEDICA, Kharkiv) monopolar in 16 loci (Fp1, Fp2, F3, F4, F7, F8, C3, C4, T3, T4, P3, P4, T5, T6, O1, $\mathrm{O} 2)$ by 10-20 international system, with the reference electrodes A and Ref tassels on the ears. The duration of the epoch was $25 \mathrm{sec}$. Among the options considered the average EEG amplitude $(\mu \mathrm{V})$, average frequency $(\mathrm{Hz})$, frequency deviation $(\mathrm{Hz})$, index $(\%)$, coefficient of asymmetry $(\%)$ and absolute $\left(\mu \mathrm{V}^{2} / \mathrm{Hz}\right)$ and relative $(\%)$ PSD of basic rhythms: $\beta(35 \div 13 \mathrm{~Hz}), \alpha(13 \div 8 \mathrm{~Hz}), \theta(8 \div 4 \mathrm{~Hz})$ and $\delta(4 \div 0,5 \mathrm{~Hz})$ in all loci, according to the instructions of the device. In addition, calculated Laterality Index (LI) for PSD each Rhythm using formula:

LI, $\%=\Sigma[200 \cdot($ Right - Left $) /($ Right + Left $)] / 8$

We calculated also for each locus Shannon's Entropy (h) of normalized PSD using Popovych's formula $[12,13]$ :

$\mathrm{h}=-\left[\mathrm{PSD} \alpha \bullet \log _{2} \mathrm{PSD} \alpha+\mathrm{PSD} \beta \bullet \log _{2} \mathrm{PSD} \beta+\mathrm{PSD} \theta \bullet \log _{2} \mathrm{PSD} \theta+\mathrm{PSD} \delta \bullet \log _{2} \mathrm{PSD} \delta\right] / \log _{2} 4$

Among hormones determined (VV Kikhtan) major adaptation hormones Cortisol, Testosterone and Triiodothyronine (by the ELISA with the use of analyzer "RT-2100C" and corresponding sets of reagents from “Алкор Био”, XEMA Co., Ltd and DRG International Inc.).

Immune status evaluated (MM Kovbasnyuk) as described in the manuals [14]. For phenotyping subpopulations of lymphocytes used the methods of rosette formation with sheep erythrocytes on which adsorbed monoclonal antibodies against receptors CD3, CD4, CD25, CD8, CD22 and CD56 from company "Granum" (Kharkiv) with visualization under light microscope with immersion system. Subpopulation of T cells with receptors high affinity (T-active) determined by test of "active" rosette formation. The state of humoral immunity judged by the concentration in serum of Circulating Immune Complexes (by polyethylene glycol precipitation method) and Immunoglobulins classes M, G, A (ELISA, analyzer "Immunochem", USA). In addition, the level of C-reactive Protein, IL-1 $\beta$ and IL-6 was determined (by the ELISA with the use of analyzer "RT-2100C" and corresponding set of reagents from "Vector-Best", RF).

Parameters of phagocytic function of neutrophils estimated as described by Douglas and Quie [15] with moderately modification by Kovbasnyuk [16,17]. The objects of phagocytosis served daily cultures of Staphylococcus aureus (ATCC N 25423 F49) as typical specimen for Gram-positive Bacteria and Escherichia coli (O55 K59) as typical representative of Gram-negative Bacteria. Both cultures obtained from Laboratory of Hydro-Geological Regime-Operational Station JSC "Truskavets'kurort". Take into account the following parameters of Phagocytosis: activity (percentage of neutrophils, in which found microbes - Hamburger's Phagocytic Index PhI), intensity (number of microbes absorbed one phagocytes - Microbial Count MC or Right's Index) and completeness (percentage of dead microbes - Killing Index KI). On the basis of the recorded partial parameters of Phagocytosis, taking into account the Neutrophils (N) content of 1 L blood, we calculated the integral parameter - Bactericidal Capacity of Neutrophils (BCCN) by the formula $[18,19]$ :

$\operatorname{BCCN}\left(10^{9}\right.$ Bacteria/L) $=\mathrm{N}\left(10^{9} / \mathrm{L}\right) \cdot \mathrm{PhI}(\%) \bullet \mathrm{MC}(\mathrm{Bact} / \mathrm{Phag}) \cdot \mathrm{KI}(\%) \bullet 10^{-4}$

We calculated also the Entropy (h) of Immunocytogram (ICG) and Leukocytogram (LCG) using Popovych's formulas [20]:

$\mathrm{hICG}=-\left[\mathrm{CD} 4 \bullet \log _{2} \mathrm{CD} 4+\mathrm{CD} 8 \bullet \log _{2} \mathrm{CD} 8+\mathrm{CD} 22 \bullet \log _{2} \mathrm{CD} 22+\mathrm{CD} 56 \bullet \log _{2} \mathrm{CD} 56\right] / \log _{2} 4$;

$\mathrm{hLCG}=-\left[\mathrm{L} \cdot \log _{2} \mathrm{~L}+\mathrm{M} \bullet \log _{2} \mathrm{M}+\mathrm{E} \bullet \log _{2} \mathrm{E}+\mathrm{PMNN} \bullet \log _{2} \mathrm{PMNN}+\mathrm{RSN} \bullet \log _{2} \mathrm{RSN}\right] / \log _{2} 5$.

Results processed by using the software package "Statistica 64".

A fragment of the work was published earlier [21]. 


\section{RESULTS}

During the week before testing, the average level of Ap-index was in the range of $7 \div 13 \mathrm{nT}$ (Fig. 1).

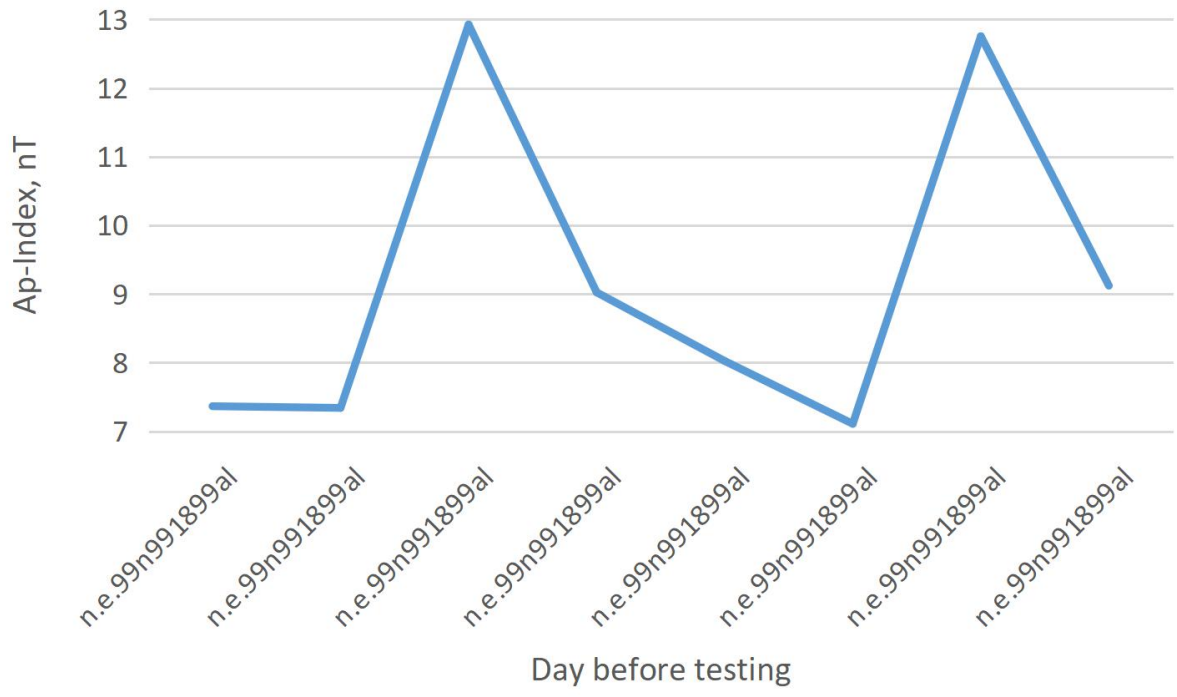

Fig. 1. Values of Ap-Index $(\mathrm{M} \pm \mathrm{SE})$ on the day of testing and during the previous 7 days

Screenings of correlations between Ap-indices and AP performed (Table 1).

Table 1. Matrix of correlations between Ap-Indices and AP conductivity

\begin{tabular}{|l|c|c|c|c|c|c|c|c|}
\hline AP & Ap-7 & Ap-6 & Ap-5 & Ap-4 & Ap-3 & Ap-2 & Ap-1 & Ap0 \\
\hline IG L & 0,15 & $\mathbf{0 , 4 0}$ & $-0,10$ & $-0,01$ & $-0,01$ & 0,04 & 0,05 & $\mathbf{0 , 1 5}$ \\
\hline Dg(Al) R & 0,12 & 0,15 & $-0,06$ & $-0,03$ & $-0,08$ & $-0,09$ & $-0,02$ & 0,13 \\
\hline GI L & 0,08 & 0,10 & $-0,03$ & 0,00 & 0,06 & $-0,16$ & $-0,01$ & 0,01 \\
\hline LY LI & 0,04 & $\mathbf{0 , 2 1}$ & $-0,12$ & 0,09 & 0,06 & 0,04 & 0,06 & 0,09 \\
\hline Dg(Al) LI & 0,04 & 0,11 & $-0,08$ & 0,00 & $-0,04$ & $-0,03$ & 0,05 & 0,08 \\
\hline IG LI & $-0,13$ & $\mathbf{- 0 , 3 6}$ & $-0,08$ & 0,05 & 0,10 & $-0,04$ & $-0,06$ & $-0,10$ \\
\hline GI LI & $-0,04$ & $-0,23$ & 0,11 & 0,09 & 0,01 & $-0,07$ & $-0,01$ & $-0,12$ \\
\hline GI R & $-0,02$ & $\mathbf{- 0 , 2 6}$ & 0,09 & 0,13 & 0,04 & $-0,16$ & $-0,02$ & $-0,16$ \\
\hline LY L & $-0,01$ & $\mathbf{- 0 , 2 0}$ & 0,12 & 0,11 & 0,05 & 0,13 & 0,00 & $-0,11$ \\
\hline SPE L & $-0,10$ & $-0,13$ & 0,09 & 0,19 & $\mathbf{0 , 1 8}$ & $-0,01$ & $-0,13$ & $\mathbf{- 0 , 3 2}$ \\
\hline TR(X) L & $-0,08$ & $\mathbf{- 0 , 1 4}$ & 0,04 & $-0,07$ & 0,00 & 0,09 & $\mathbf{0 , 1 5}$ & 0,00 \\
\hline MC(AVL) LI & $-0,09$ & $\mathbf{- 0 , 2 2}$ & $-0,16$ & $-\mathbf{0 , 2 3}$ & $\mathbf{- 0 , 2 2}$ & $-0,05$ & $\mathbf{0 , 3 4}$ & 0,15 \\
\hline IG R & $-0,02$ & $-0,12$ & $-0,01$ & $-\mathbf{0 , 1 6}$ & $-0,11$ & 0,07 & $\mathbf{0 , 2 4}$ & $\mathbf{0 , 1 5}$ \\
\hline Pg(ND) LI & 0,08 & $-0,02$ & 0,02 & $-0,04$ & $-0,02$ & 0,04 & $\mathbf{0 , 1 9}$ & $\mathbf{0 , 1 4}$ \\
\hline Pg(ND) R & $-0,05$ & $-0,06$ & 0,06 & 0,08 & $\mathbf{0 , 1 8}$ & 0,16 & $-0,01$ & $\mathbf{- 0 , 1 6}$ \\
\hline Pg(ND) L & $-0,08$ & $-0,05$ & 0,06 & 0,10 & 0,19 & 0,14 & $-0,09$ & $\mathbf{- 0 , 2 2}$ \\
\hline MC(AVL) R & $-0,08$ & $-0,14$ & $-0,06$ & $-0,08$ & $-0,02$ & 0,09 & $\mathbf{0 , 2 3}$ & 0,02 \\
\hline SPE LI & 0,07 & 0,14 & $-0,04$ & $-0,13$ & $-0,16$ & 0,08 & 0,07 & $\mathbf{0 , 2 3}$ \\
\hline LY R & 0,05 & 0,04 & 0,07 & $\mathbf{0 , 1 6}$ & 0,08 & 0,14 & 0,07 & 0,10 \\
\hline C(V) R & $-0,03$ & 0,01 & 0,08 & $-0,06$ & $-0,09$ & 0,06 & 0,06 & 0,13 \\
\hline C(V) L & $-0,07$ & $-0,05$ & $-0,03$ & $-0,05$ & $-0,02$ & $-0,05$ & 0,00 & $-0,01$ \\
\hline C(V) LI & 0,06 & 0,05 & 0,05 & 0,01 & $-0,02$ & 0,05 & 0,01 & 0,05 \\
\hline Dg(Al) L & $-0,06$ & $-0,09$ & $-0,02$ & $-0,02$ & $-0,02$ & 0,02 & $-0,02$ & $-0,05$ \\
\hline SPE R & 0,01 & $-0,00$ & $-0,05$ & 0,14 & $-0,06$ & 0,05 & $-0,10$ & 0,05 \\
\hline MC(AVL) L & $-0,05$ & $-0,06$ & 0,01 & 0,02 & 0,08 & 0,12 & 0,09 & $-0,05$ \\
\hline TR(X) R & $-0,06$ & $-0,14$ & 0,02 & $-0,12$ & $-0,03$ & 0,08 & 0,17 & 0,04 \\
\hline TR(X) LI & 0,05 & 0,01 & $-0,07$ & $-0,12$ & $-0,09$ & $-0,04$ & 0,04 & 0,11 \\
\hline Multiple R & 0,169 & $\mathbf{0 , 5 5 2}$ & 0,192 & $\mathbf{0 , 3 4 8}$ & $\mathbf{0 , 3 4 0}$ & 0,238 & $\mathbf{0 , 4 2 6}$ & $\mathbf{0 , 4 7 0}$ \\
\hline No. Accoring & (N) & \\
\hline
\end{tabular}

Note. According to calculations by the formula: $|r|=\left\{\exp \left[2 \mathrm{t} /(\mathrm{n}-1,5)^{0,5}\right]-1\right\} /\left\{\exp \left[2 \mathrm{t} /(\mathrm{n}-1,5)^{0,5}\right]+1\right\}$ 
for a sample of $\mathrm{n}=82$ critical value $|\mathrm{r}|$ at $\mathrm{p}<0,05(\mathrm{t}>2,00)$ is 0,22 , at $\mathrm{p}<0,01(\mathrm{t}>2,66)$ is 0,29 , at $\mathrm{p}<0,001(\mathrm{t}>3,46)$ is 0,37 . The coefficients included in the regression models are marked in bold.

Construction of regression models by stepwise exclusion to reach the maximum value of Adjusted $\mathrm{R}^{2}$ found that the electrical conductivity of APs and its symmetry is most closely correlated with Ap-index 6 days before registration (Table 2 and Fig. 7). The pronounced asymmetry of the effect of Ap-index on the electrical conductivity of AP IG is noteworthy: upregulating to the left point instead of downregulating to the right.

Table 2. Regression Summary for Ap-6

$\mathrm{R}=0,552 ; \mathrm{R}^{2}=0,305 ;$ Adjusted $\mathrm{R}^{2}=0,259 ; \mathrm{F}_{(5,8)}=6,7 ; \mathrm{p}<10^{-4}$

\begin{tabular}{|l|l|l|l|l|l|l|l|}
\hline \multicolumn{2}{|l|}{} & Beta & $\begin{array}{l}\text { St. Err. } \\
\text { of Beta }\end{array}$ & B & $\begin{array}{l}\text { St. Err. } \\
\text { of B }\end{array}$ & $\mathrm{t}_{(76)}$ & $\begin{array}{l}\text { p- } \\
\text { level }\end{array}$ \\
\hline Variables & r & & Intercpt & $-42,4$ & 37,8 & $-1,12$ & 0,266 \\
\hline IG Left, units & $\mathbf{0 , 4 0}$ & $-0,244$ & 0,105 & $-0,520$ & 0,224 & $-2,32$ & 0,023 \\
\hline IG Laterality Index, \% & $\mathbf{- 0 , 3 6}$ & 1,621 & 0,554 & 1,571 & 0,537 & 2,93 & 0,005 \\
\hline GI Right, units & $\mathbf{- 0 , 2 6}$ & 0,481 & 0,108 & 0,00009 & 0,00002 & 4,44 & $10^{-4}$ \\
\hline MC(AVL) LI, \% & $\mathbf{- 0 , 2 2}$ & 1,331 & 0,555 & 0,703 & 0,293 & 2,40 & 0,019 \\
\hline TR(X) Right, units & $\mathbf{- 0 , 1 4}$ & $-0,234$ & 0,099 & $-58,81$ & 24,91 & $-2,36$ & 0,021 \\
\hline
\end{tabular}

According to the analysis of the canonical correlation between Ap-indices and AP-conductivity, it was found (Table 3) that the geomagnetic root receives the maximum factor load from the Ap-Index 6 days before testing. Much less, but unidirectional load on the root gives Ap-Index 4 and 3 days before the registration of conductivity. Instead, the Ap-Index on the eve of the measurement gives the load with the opposite sign. Apindexes on other days give meager factor loads and were not included by the program in the model.

The effective (dependent) root is represented, on the one hand, by APs whose electrical conductivity increases under the influence of the Ap-index 6 days before registration or its lateralization shifts to the right, and on the other hand, APs whose symmetry shifts to the left or whose electrical conductivity decreases. The third constellation is represented by three parameters that are upregulated by Ap-1 instead downregulated by Ap-indices on other days.

In general, the electrical conductivity of these APs or its symmetry is determined by Ap-indices by $44 \%$ (Fig. 2).

Table 3. Factor Structure Matrix for Canonical Roots of Ap-Indices and AP conductivity

\begin{tabular}{|l|l|}
\hline Left set & $\mathbf{R}$ \\
\hline Ap-6, nT & $\mathbf{- 0 , 7 6 5}$ \\
\hline Ap-4, nT & $\mathbf{- 0 , 3 0 8}$ \\
\hline Ap-3, nT & $\mathbf{- 0 , 1 4 4}$ \\
\hline Ap-1, nT & $\mathbf{0 , 4 9 4}$ \\
\hline Right set & $\mathbf{R}$ \\
\hline IG left & $\mathbf{- 0 , 5 2 7}$ \\
\hline Dg(Al) right & $\mathbf{- 0 , 2 3 4}$ \\
\hline GI left & $\mathbf{- 0 , 1 5 3}$ \\
\hline LY LI & $\mathbf{- 0 , 3 0 5}$ \\
\hline Dg(Al) LI & $\mathbf{- 0 , 1 7 9}$ \\
\hline IG LI & $\mathbf{0 , 4 8 0}$ \\
\hline GI LI & $\mathbf{0 , 2 5 6}$ \\
\hline LY left & $\mathbf{0 , 2 6 0}$ \\
\hline GI right & $\mathbf{0 , 2 4 0}$ \\
\hline SPE left & $\mathbf{0 , 1 6 9}$ \\
\hline TR(X) left & $\mathbf{0 , 3 0 6}$ \\
\hline MC(AVL) LI & $\mathbf{0 , 4 2 2}$ \\
\hline Pg(ND) LI & $\mathbf{0 , 2 1 4}$ \\
\hline IG right & $\mathbf{0 , 2 9 3}$ \\
\hline
\end{tabular}




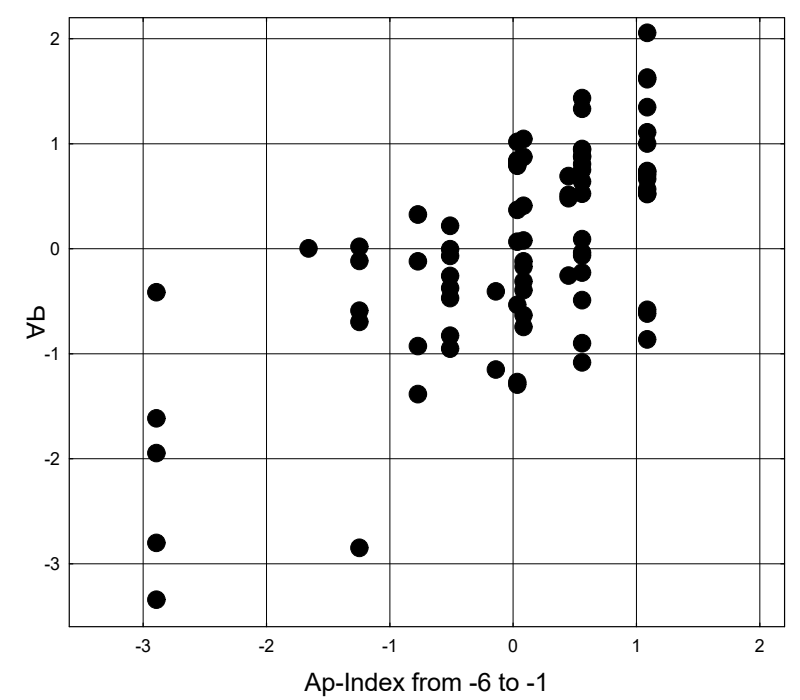

$\mathrm{R}=0,661 ; \mathrm{R}^{2}=0,437 ; \chi^{2}{ }_{(84)}=108 ; \mathrm{p}=0,038 ; \Lambda$ Prime $=0,215$

Fig. 2. Scatterplot of canonical correlation between Ap Geomagnetic indices during 6 days before testing (X-line) and electrical conductivity of Acupuncture Points (Y-line)

Based on the analysis of the canonical correlation between the electrical conductivity of 9 AP pairs and their symmetry, on the one hand, and the amplitude-frequency and spectral parameters of EEG, on the other hand, two pairs of canonical roots were identified.

AP-root of the first pair (table. 4) represent 4 parameters directly and one - in reverse. EEG-root parameters are subjected to pseudo-staining for clarity. It is clear that 13 spectral parameters of theta rhythm, as well as entropy PSD in locus P3 with negative factor loads are inversely related to the electrical conductivity of AP IG right. On the other hand, the amplitude and index of the alpha rhythm, as well as its 12 spectral parameters, are directly related to the electrical conductivity of AP LY left, which is also inversely related to the parameters of the theta rhythm.

The conductivity lateralization indices in the LY and $\mathrm{Dg}(\mathrm{Al})$ pairs correlate positively with the three beta-rhythm parameters, in addition to the positive relationships with the theta-rhythm parameters and the negative relationships with the alpha-rhythm parameters. Finally, AP TR (X) left has a positive relationship only with PSD T3- $\beta$. The canonical correlation between the two sets is drastically strong (Fig. 2).

Table 4. Factor Structure Matrix for first pair APs/EEG Canonical Roots

\begin{tabular}{|c|c|}
\hline Left set & R1 \\
\hline IG right & 0,995 \\
\hline LY left & $-0,780$ \\
\hline LY Laterality Index & 0,558 \\
\hline Dg(Al) Laterality Ind & 0,335 \\
\hline TR(X) left & 0,195 \\
\hline Right set & R1 \\
\hline PSD P3- $\theta, \%$ & $-0,932$ \\
\hline PSD P4- $\theta, \%$ & $-0,788$ \\
\hline PSD P4- $\theta, \mu V^{2} / H z$ & $-0,618$ \\
\hline PSD C $3-\theta, \%$ & $-0,754$ \\
\hline PSD 01- $\theta, \%$ & $-0,749$ \\
\hline PSD 01- $\theta, \mu V^{2} / H z$ & $-0,626$ \\
\hline PSD 02- $\theta, \%$ & $-0,724$ \\
\hline PSD C $4-\theta, \%$ & $-0,653$ \\
\hline PSD T6- $\theta, \%$ & $-0,641$ \\
\hline PSD F7- $\theta, \%$ & $-0,618$ \\
\hline PSD F4- $\theta, \%$ & $-0,610$ \\
\hline PSD T5- $\theta, \%$ & $-0,575$ \\
\hline PSD F3- $\theta, \%$ & $-0,546$ \\
\hline Entropy PSD P3 & $-0,440$ \\
\hline
\end{tabular}




\begin{tabular}{|c|c|}
\hline PSD 02- $\alpha, \%$ & $-0,719$ \\
\hline PSD P4- $\alpha, \%$ & $-0,586$ \\
\hline PSD T6- $\alpha, \%$ & $-0,572$ \\
\hline PSD 01- $\alpha, \%$ & $-0,554$ \\
\hline PSD F4- $\alpha, \%$ & $-0,509$ \\
\hline PSD C $4-\alpha, \%$ & $-0,506$ \\
\hline Index $\alpha, \%$ & $-0,482$ \\
\hline PSD Fp2- $\alpha, \%$ & $-0,470$ \\
\hline PSD F3- $\alpha, \%$ & $-0,465$ \\
\hline PSD P3- $\alpha, \%$ & $-0,464$ \\
\hline PSD C $3-\alpha, \%$ & $-0,438$ \\
\hline PSD T5- $\alpha, \%$ & $-0,327$ \\
\hline PSD O2- $\alpha, \mu V^{2} / H z$ & $-0,322$ \\
\hline Amplitude $\alpha, \mu V$ & $-0,245$ \\
\hline PSD T3- $\beta, \mu V^{2} / H z$ & 0,332 \\
\hline PSD O2- $\beta, \%$ & 0,317 \\
\hline PSD P4- $\beta, \%$ & 0,239 \\
\hline
\end{tabular}

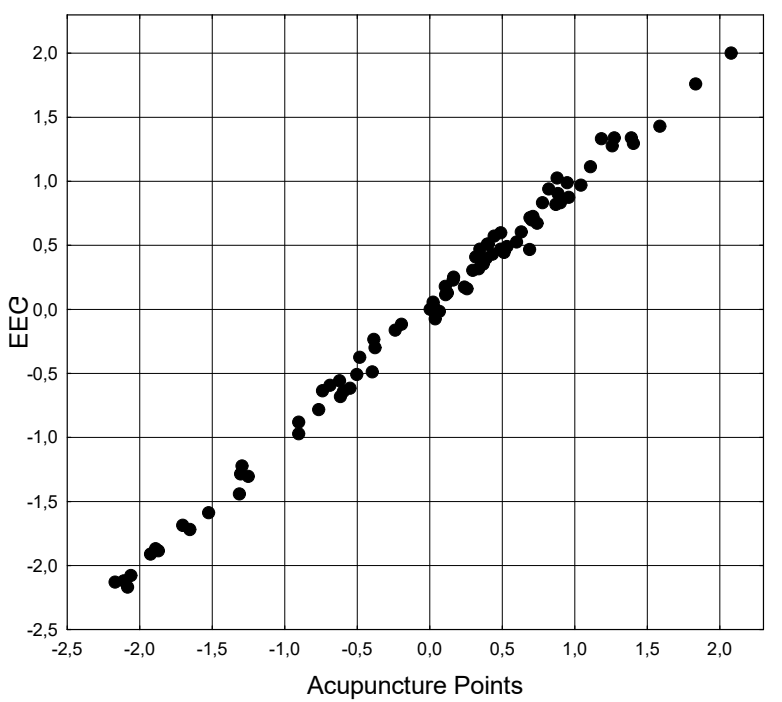

$\mathrm{R}=0,997 ; \mathrm{R}^{2}=0,994 ; \chi^{2}{ }_{(280)}=944 ; \mathrm{p}<10^{-6} ; \Lambda$ Prime $<10^{-6}$

Fig. 3. Scatterplot of canonical correlation between Acupuncture Points (X-line) and EEGs (Y-line) parameters. First pair of Roots

The AP-root of the second pair (Table 5) presents only three parameters. On the other hand, EEG-root represents, first of all, 18 parameters of alpha rhythm, as well as lateralization of beta rhythm, which are related to the electrical conductivity of AP LY left directly, while its lateralization is inverse. The spectral parameters of beta-rhythm, as well as entropy PSD in locus P3 correlate positively with the lateralization of electrical conductivity in pairs LY and Dg (Al), while negatively - with the electrical conductivity AP LY left. The canonical correlation between these two sets is somewhat weaker, but also impressive (Fig. 3). 
Table 5. Factor Structure Matrix for second pair APs/EEG Canonical Roots

\begin{tabular}{|c|c|}
\hline Left set & $\mathbf{R 2}$ \\
\hline LY left & 0,506 \\
\hline LY Laterality Index & $-0,497$ \\
\hline $\operatorname{Dg}(\mathrm{Al})$ Laterality Ind & $-0,312$ \\
\hline Right set & $\mathbf{R 2}$ \\
\hline PSD T5- $\alpha, \mu V^{2} / H z$ & 0,524 \\
\hline PSD T5- $\alpha, \%$ & $\mathbf{0 , 5 2 0}$ \\
\hline PSD P3- $\alpha, \mu V^{2} / H z$ & 0,523 \\
\hline PSD P3- $\alpha, \%$ & 0,492 \\
\hline Index $\alpha, \%$ & 0,506 \\
\hline Amplitude $\alpha, \mu \mathrm{V}$ & 0,478 \\
\hline PSD 01- $\alpha, \mu V^{2} / H z$ & 0,482 \\
\hline PSD 01- $\alpha, \%$ & $\mathbf{0 , 4 5 2}$ \\
\hline PSD P4- $\alpha, \mu V^{2} / H z$ & 0,472 \\
\hline PSD P4- $\alpha, \%$ & 0,406 \\
\hline PSD O2- $\alpha, \mu V^{2} / H z$ & 0,414 \\
\hline PSD 02- $\alpha, \%$ & 0,343 \\
\hline PSD T6- $\alpha, \%$ & 0,375 \\
\hline PSD C $3-\alpha, \%$ & 0,356 \\
\hline PSD F4- $\alpha, \%$ & $\mathbf{0 , 3 3 8}$ \\
\hline PSD C4- $\alpha, \%$ & $\mathbf{0 , 3 3 5}$ \\
\hline PSD Fp2- $\alpha, \%$ & 0,329 \\
\hline PSD F3- $\alpha, \%$ & 0,316 \\
\hline Laterality $\beta, \%$ & $\mathbf{0 , 3 6 7}$ \\
\hline PSD $\mathrm{T} 3-\beta, \mu \mathrm{V}^{2} / \mathrm{Hz}$ & $-0,589$ \\
\hline PSD P4- $\beta, \%$ & $-0,484$ \\
\hline PSD 02- $\beta, \%$ & $-0,340$ \\
\hline Entropy PSD P3 & $-0,324$ \\
\hline
\end{tabular}

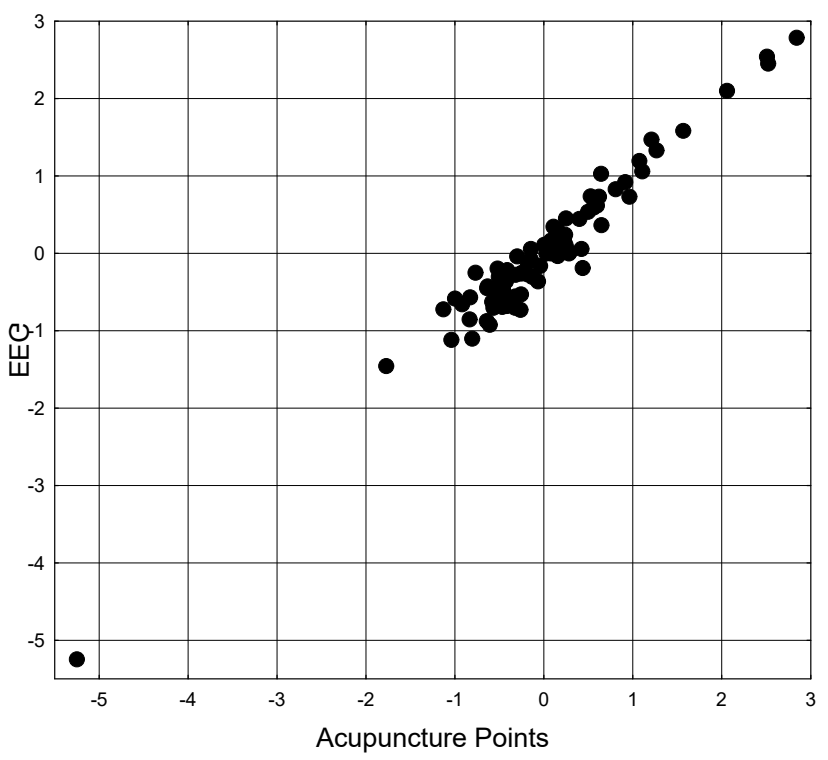

$\mathrm{R}=0,978 ; \mathrm{R}^{2}=0,956 ; \chi^{2}(238)=645 ; \mathrm{p}<10^{-6} ; \Lambda$ Prime $<10^{-5}$

Fig. 4. Scatterplot of canonical correlation between Acupuncture Points (X-line) and EEGs (Y-line) parameters. Second pair of Roots

Next, following the accepted algorithm, consider the matrix of correlations between AP parameters, on the one hand, and HRV and endocrine parameters - on the other hand. Pseudo-coloring visualizes several constellations of connections. First, the electrical conductivity of AP GI left and Dg(Al) right, as well as the 
lateralization of the latter and LY are negatively correlated with HRV-markers of vagal tone and current plasma levels of testosterone and triiodothyronine, while positively with HRV-markers of sympathetic tone and levels in plasma cortisol and testosterone, standardized by sex and age. Second, the electrical conductivity of AP IG right, LY left, GI right and lateralization of the latter pair, in contrast, correlate positively with HRV-markers of vagal tone and current testosterone levels, while negatively - with HRV-markers of sympathetic tone and standardized testosterone levels. Finally, the electrical conductivity of AP TR(X) left again has only one positive relationship - with triiodothyronine.

Table 6. Matrix of correlations between selected APs conductivity and HRV/Hormonal variables

\begin{tabular}{|c|c|c|c|c|c|c|c|c|c|}
\hline Variables & $\begin{array}{l}\mathrm{Dg}(\mathrm{Al}) \\
\mathrm{r}\end{array}$ & $\begin{array}{l}\text { Dg(Al) } \\
\text { LI }\end{array}$ & $\begin{array}{l}\text { LY } \\
\text { LI }\end{array}$ & $\begin{array}{l}\text { GI } \\
1\end{array}$ & $\begin{array}{l}\text { GI } \\
\mathrm{r}\end{array}$ & $\begin{array}{l}\text { GI } \\
\text { LI }\end{array}$ & $\begin{array}{l}\mathrm{LY} \\
\mathrm{l}\end{array}$ & $\begin{array}{l}\text { IG } \\
\mathrm{r}\end{array}$ & $\begin{array}{l}\text { TR(X) } \\
1\end{array}$ \\
\hline HF PS, $\mathrm{msec}^{2}$ & $-0,31$ & $-0,10$ & $-0,13$ & $-0,17$ & 0,27 & 0,27 & 0,17 & 0,29 & $-0,16$ \\
\hline LF PS, $\mathrm{msec}^{2}$ & $-0,28$ & $-0,11$ & $-0,19$ & $-0,12$ & 0,24 & 0,23 & 0,23 & 0,28 & $-0,02$ \\
\hline Total Power, $\mathrm{msec}^{2}$ & $-0,21$ & $-0,20$ & $-0,12$ & $-0,09$ & 0,16 & 0,16 & 0,17 & 0,18 & $-0,03$ \\
\hline SDNN, msec & $-0,21$ & $-0,18$ & $-0,18$ & $-0,09$ & 0,22 & 0,20 & 0,22 & 0,23 & $-0,08$ \\
\hline VLF PS, $\mathrm{msec}^{2}$ & $-0,15$ & $-0,22$ & $-0,08$ & $-0,09$ & 0,09 & 0,11 & 0,11 & 0,12 & $-0,02$ \\
\hline Triangular Index, units & $-0,14$ & $-0,05$ & $-0,22$ & $-0,08$ & 0,25 & 0,22 & 0,23 & 0,19 & $-0,05$ \\
\hline $\mathrm{pNN}_{50}, \%$ & $-0,15$ & $-0,07$ & $-0,15$ & $-0,18$ & 0,29 & 0,29 & 0,17 & 0,24 & $-0,18$ \\
\hline RMSSD, msec & $-0,14$ & $-0,10$ & $-0,16$ & $-0,18$ & 0,30 & 0,30 & 0,20 & 0,30 & $-0,20$ \\
\hline $\mathrm{HF} / \mathrm{TP}, \%$ & $-0,09$ & $-0,16$ & $-0,01$ & $-0,14$ & 0,14 & 0,17 & 0,07 & 0,29 & $-0,19$ \\
\hline Heart Rate, beats/min & 0,03 & $-0,02$ & 0,21 & 0,09 & $-0,23$ & $-0,21$ & $-0,11$ & 0,01 & 0,10 \\
\hline Baevsky's Stress Ind, ln & 0,11 & 0,01 & 0,20 & 0,06 & $-0,23$ & $-0,19$ & $-0,19$ & $-0,18$ & 0,07 \\
\hline Amplitude of Mode, $\%$ & 0,11 & 0,02 & 0,20 & 0,10 & $-0,22$ & $-0,20$ & $-0,21$ & $-0,21$ & 0,10 \\
\hline $\mathrm{LF} /(\mathrm{LF}+\mathrm{HF}), \%$ & 0,07 & 0,07 & 0,04 & 0,15 & $-0,19$ & $-0,21$ & $-0,03$ & $-0,22$ & 0,20 \\
\hline ULF/TP, \% & 0,06 & 0,05 & $-0,01$ & 0,25 & $-0,00$ & $-0,11$ & 0,01 & $-0,22$ & 0,08 \\
\hline Testosterone, $\mathrm{nM} / \mathrm{L}$ & $-0,27$ & $-0,08$ & $-0,10$ & $-0,16$ & 0,33 & 0,31 & 0,12 & $-0,13$ & 0,04 \\
\hline Triiodothyronine, $\mathrm{nM} / \mathrm{L}$ & $-0,26$ & $-0,06$ & $-0,02$ & 0,07 & 0,15 & 0,08 & 0,01 & $-0,16$ & 0,22 \\
\hline Cortisol, nM/L & 0,14 & 0,07 & 0,25 & 0,13 & $-0,15$ & $-0,17$ & $-0,18$ & $-0,24$ & 0,11 \\
\hline Testosterone standard, Z & 0,12 & 0,07 & 0,19 & 0,03 & $-0,16$ & $-0,13$ & $-0,18$ & $-0,17$ & $-0,12$ \\
\hline
\end{tabular}

The canonical roots formed on the basis of the correlation matrix indicate that the relationship between AP and HRV\&Hormonal parameters is also very strong (Table 7 and Fig. 5).

Table 7. Factor Structure Matrix for APs/HRV\&Hormonal Canonical Roots

\begin{tabular}{|c|c|}
\hline Left set & $\mathbf{R}$ \\
\hline $\mathrm{Dg}(\mathrm{Al})$ right & $-0,850$ \\
\hline LY LI & $-0,198$ \\
\hline $\operatorname{Dg}(\mathrm{Al}) \mathrm{LI}$ & $-0,190$ \\
\hline GI left & $-0,158$ \\
\hline GI right & 0,508 \\
\hline GI LI & 0,443 \\
\hline LY left & 0,218 \\
\hline IG right & 0,100 \\
\hline TR(X) left & 0,306 \\
\hline Right set & $\mathbf{R}$ \\
\hline HF band PS, $\mathrm{msec}^{2}$ & 0,283 \\
\hline LF band PS, $\mathrm{msec}^{2}$ & 0,272 \\
\hline Total Power Spectral, $\mathrm{msec}^{2}$ & 0,193 \\
\hline SDNN, msec & 0,167 \\
\hline VLF band PS, $\mathrm{msec}^{2}$ & 0,129 \\
\hline Triangular Index, units & 0,122 \\
\hline $\mathrm{pNN}_{50}, \%$ & 0,109 \\
\hline RMSSD, msec & 0,058 \\
\hline Heart Rate, beats/min & $-0,089$ \\
\hline Baevsky's Stress Index, ln & $-0,088$ \\
\hline
\end{tabular}




\begin{tabular}{|l|c|}
\hline Testosterone actual, $\mathrm{nM} / \mathrm{L}$ & 0,517 \\
\hline Testosterone standardized, $\mathrm{Z}$ & $-0,104$ \\
\hline Cortisol, $\mathrm{nM} / \mathrm{L}$ & $-0,029$ \\
\hline Triiodothyronine, $\mathrm{nM} / \mathrm{L}$ & $\mathbf{0 , 5 1 5}$ \\
\hline
\end{tabular}

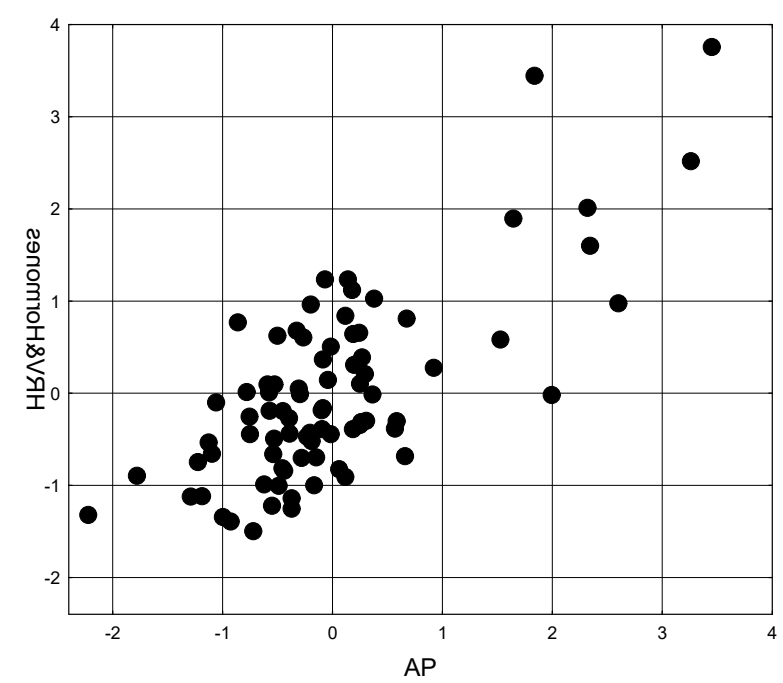

$\mathrm{R}=0,740 ; \mathrm{R}^{2}=0,548 ; \chi^{2}(126)=156 ; \mathrm{p}=0,035 ; \Lambda$ Prime $=0,104$

Fig. 5. Scatterplot of canonical correlation between Acupuncture Points (X-line) and HRV\&Hormonal (Y-line) parameters

Analysis of the canonical correlation between Ap-Indices and EEG parameters (Table 8) shows the greatest sensitivity to geomagnetic perturbations of theta rhythm. Interestingly, on the day and on the eve of EEG registration geomagnetic disturbances have an inhibitory effect, while 3 and 4 days before it - activating.

Only after five days does the delta rhythm in the F8, T6, and Fp1 loci (activation) and the beta rhythm in general and the $\mathrm{O} 2$ locus in particular (suppression) respond to geomagnetic disturbances. An even longer stimulation lag was found for the beta rhythm at the F3 and $\mathrm{C} 3$ loci as well as the theta rhythm at the T5 locus, whereas the delta rhythm at the F7 locus is currently suppressed.

We would like to separately note the positive correlation of Entropy in locus O2 7 days before registration, which is inverted every other day.

Table 8. Matrix of correlations between Ap-Indices and EEG parameters

\begin{tabular}{|c|c|c|c|c|c|c|c|c|}
\hline Variables & Ap-7 & Ap-6 & Ap-5 & Ap-4 & Ap-3 & Ap-2 & Ap-1 & Ap0 \\
\hline PSD C4- $\theta, \%$ & 0,09 & 0,10 & 0,05 & 0,25 & 0,28 & 0,06 & $-0,30$ & $-0,30$ \\
\hline PSD C $3-\theta, \%$ & 0,01 & 0,05 & 0,10 & 0,25 & 0,25 & 0,08 & $-0,28$ & $-0,28$ \\
\hline PSD T6- $\theta, \%$ & $-0,03$ & 0,15 & 0,03 & 0,25 & 0,23 & 0,07 & $-0,29$ & $-0,26$ \\
\hline PSD C4- $\theta, \mu V^{2} / H z$ & 0,02 & $-0,00$ & $-0,16$ & $-0,05$ & 0,01 & $-0,18$ & $-0,27$ & $-0,26$ \\
\hline PSD T4- $\theta, \%$ & 0,16 & 0,10 & $-0,11$ & 0,14 & 0,20 & 0,01 & $-0,26$ & $-0,23$ \\
\hline PSD F7- $\theta, \%$ & 0,12 & 0,19 & $-0,11$ & 0,13 & 0,21 & $-0,05$ & $-0,30$ & $-0,21$ \\
\hline PSD F8- $\theta, \%$ & $-0,06$ & $-0,04$ & $-0,20$ & 0,06 & 0,06 & $-0,07$ & $-0,19$ & $-0,27$ \\
\hline PSD F4- $\theta, \mu V^{2} / H z$ & $-0,03$ & 0,06 & $-0,04$ & $-0,00$ & 0,07 & $-0,14$ & $-0,27$ & $-0,22$ \\
\hline PSD F4- $\theta, \%$ & 0,03 & 0,02 & 0,02 & 0,19 & 0,18 & 0,08 & $-0,24$ & $-0,25$ \\
\hline PSD Fp1- $\theta, \%$ & $-0,05$ & 0,13 & $-0,08$ & 0,11 & 0,09 & 0,02 & $-0,26$ & $-0,16$ \\
\hline PSD P4- $\theta, \mu V^{2} / H z$ & $-0,01$ & $-0,02$ & $-0,11$ & 0,07 & 0,09 & $-0,07$ & $-0,20$ & $-0,25$ \\
\hline PSD T6- $\alpha, \mu \mathrm{V}^{2} / \mathrm{Hz}$ & $-0,10$ & $-0,18$ & $-0,13$ & $-0,02$ & $-0,02$ & $-0,11$ & $-0,13$ & $-0,25$ \\
\hline PSD $02-\theta, \%$ & 0,15 & 0,14 & $-0,10$ & 0,18 & 0,23 & 0,08 & $-0,15$ & $-0,17$ \\
\hline PSD F8 $\delta, \mu V 2 / H z$ & 0,08 & 0,02 & 0,30 & 0,15 & 0,15 & 0,09 & $-0,14$ & $-0,06$ \\
\hline PSD T $6-\delta, \mu \mathrm{V} 2 / \mathrm{Hz}$ & $-0,05$ & $-0,04$ & 0,27 & 0,09 & 0,07 & 0,06 & $-0,07$ & $-0,04$ \\
\hline PSD Fp1- $\delta, \mu \mathrm{V} 2 / \mathrm{Hz}$ & $-0,01$ & $-0,02$ & 0,21 & 0,04 & 0,04 & 0,03 & $-0,06$ & $-0,03$ \\
\hline PSD O2- $\beta, \mu \mathrm{V} 2 / \mathrm{Hz}$ & $-0,05$ & $-0,07$ & $-0,26$ & $-0,20$ & $-0,20$ & $-0,14$ & 0,07 & 0,04 \\
\hline Amplitude $\beta, \mu \mathrm{V}$ & 0,11 & 0,10 & $-0,23$ & $-0,17$ & $-0,10$ & $-0,08$ & 0,11 & 0,15 \\
\hline Entropy PSD O2 & 0,21 & 0,15 & $-0,24$ & 0,02 & 0,06 & $-0,01$ & $-0,03$ & 0,00 \\
\hline PSD F3- $\beta, \mu V 2 / \mathrm{Hz}$ & 0,26 & 0,29 & $-0,20$ & $-0,15$ & 0,02 & $-0,07$ & 0,06 & 0,18 \\
\hline
\end{tabular}




\begin{tabular}{|l|c|c|c|c|c|c|c|c|}
\hline PSD F3- $\boldsymbol{\beta}, \boldsymbol{\%}$ & $\mathbf{0 , 2 3}$ & $\mathbf{0 , 2 4}$ & $-0,13$ & $-0,02$ & 0,03 & $-0,04$ & 0,02 & 0,18 \\
\hline PSD C3- $\boldsymbol{\beta}, \boldsymbol{\%}$ & 0,14 & $\mathbf{0 , 2 7}$ & $-0,18$ & $-0,14$ & $-0,04$ & $-0,03$ & $\mathbf{0 , 2 4}$ & $\mathbf{0 , 3 0}$ \\
\hline PSD T5- $\boldsymbol{\theta}, \boldsymbol{\%}$ & 0,17 & $\mathbf{0 , 2 7}$ & $-0,04$ & 0,06 & 0,17 & $-0,03$ & $-0,14$ & $-0,00$ \\
\hline PSD F7- $\boldsymbol{\delta}, \boldsymbol{\%}$ & $-0,15$ & $-\mathbf{0 , 2 2}$ & 0,02 & $-0,06$ & $-0,05$ & 0,13 & 0,12 & $-0,06$ \\
\hline Multiple correlation R & $\mathbf{0 , 3 9 0}$ & $\mathbf{0 , 7 1 3}$ & $\mathbf{0 , 6 7 0}$ & $\mathbf{0 , 5 3 9}$ & $\mathbf{0 , 4 8 2}$ & 0,294 & $\mathbf{0 , 7 3 6}$ & $\mathbf{0 , 6 8 7}$ \\
\hline
\end{tabular}

It appears that neurons that generate different rhythms and that are projected onto different loci of the scalp respond to geomagnetic perturbation asynchronously, with a delay of 7 to 0,5 days and inversion of stimulation into inhibition.

According to the canonical correlation analysis, geomagnetic perturbations on the day of EEG registration and for seven days before it determines the EEG parameters by $79 \%$ (Table 9 and Fig. 6).

Table 9. Factor Structure Matrix for Canonical Roots of Ap-Indices and EEGs parameters

\begin{tabular}{|c|c|}
\hline Left set & $\mathbf{R}$ \\
\hline Ap-1, nT & $-0,252$ \\
\hline Ap- $0, n T$ & $-0,095$ \\
\hline Ap- $2, n T$ & $-0,068$ \\
\hline Ap-5, nT & $-0,667$ \\
\hline Ap-4, nT & $-0,293$ \\
\hline Ap-3, nT & $-0,022$ \\
\hline Ap-7, nT & 0,264 \\
\hline Ap-6, nT & 0,144 \\
\hline Right set & $\mathbf{R}$ \\
\hline PSD F7- $\theta, \%$ & 0,510 \\
\hline PSD F8- $\theta, \%$ & 0,440 \\
\hline PSD C4- $\theta, \mu V^{2} / H z$ & 0,431 \\
\hline PSD C4- $\theta, \%$ & 0,228 \\
\hline PSD Fp1- $\theta, \%$ & 0,342 \\
\hline PSD F4- $\theta, \mu V^{2} / H z$ & 0,337 \\
\hline PSD F4- $\theta, \%$ & 0,176 \\
\hline PSD P4- $\theta, \mu V^{2} / H z$ & 0,320 \\
\hline PSD T $6-\theta, \%$ & 0,223 \\
\hline PSD O2- $\theta, \%$ & 0,316 \\
\hline PSD C3- $\theta, \%$ & 0,132 \\
\hline PSD T4- $\theta, \%$ & 0,130 \\
\hline PSD T6- $\alpha, \mu V^{2} / H z$ & 0,235 \\
\hline PSD T6- $\delta, \mu \mathrm{V}^{2} / \mathrm{Hz}$ & $-0,288$ \\
\hline PSD F8- $\delta, \mu V^{2} / H z$ & $-0,263$ \\
\hline PSD Fp1- $\delta, \mu V^{2} / H z$ & $-0,218$ \\
\hline Entropy PSD O2 & 0,351 \\
\hline Amplitude $\beta, \mu \mathrm{V}$ & 0,249 \\
\hline PSD O2- $\beta, \mu V^{2} / H z$ & 0,240 \\
\hline PSD F3- $\beta, \mu V^{2} / H z$ & 0,361 \\
\hline PSD F3- $\beta, \%$ & 0,206 \\
\hline PSD C3- $\beta, \%$ & 0,162 \\
\hline PSD T5- $\theta, \%$ & 0,321 \\
\hline PSD F7- $\delta, \%$ & $-0,121$ \\
\hline
\end{tabular}




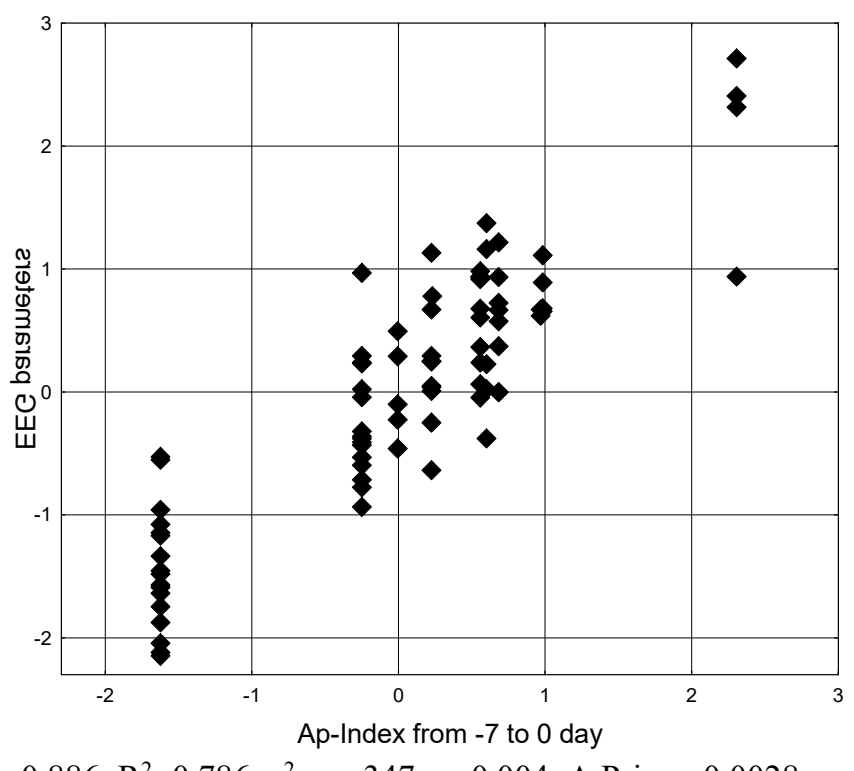

$\mathrm{R}=0,886 ; \mathrm{R}^{2}=0,786 ; \chi^{2}(280)=347 ; \mathrm{p}=0,004 ; \Lambda$ Prime $=0,0028$

Fig. 6. Scatterplot of canonical correlation between Ap Geomagnetic indices before 7 days and on day testing (X-line) and EEG parameters (Y-line)

Color coding of rhythms immediately suggests the association of the limits of their ranges with Schuman's resonance harmonics: the first $(8,5$ and $7,83 \mathrm{~Hz})$ and second $(14,7$ and $14,1 \mathrm{~Hz})$ (author's and experimentally established respectively). More about this in the discussion.

Given the close relationship between the central and autonomic nervous systems, as well as both with the endocrine system, the matrix of correlations between Ap-Indices and HRV\&Endocrine parameters was created (Table 10).

From the above it seems that geomagnetic disturbances with a lag of 7-5 days cause a sympathotonic shift of the autonomic balance due, first of all, to a decrease in vagal tone and, to a lesser extent, an increase in sympathetic tone and circulating catecholamines (marked by 1/Mode ratio).

With a two-day delay, ULF band (the physiological essence of which still remains as terra incognita) upregulated by geomagnetic disturbances, while plasma testosterone levels (not relevant, but standardized by sex and age of patients) downregulated. Triiodothyronine and cortisol downregulated by geomagnetic disturbances on the day of blood collection.

Table 10. Matrix of correlations between Ap-Indices and HRV\&Endocrine parameters

\begin{tabular}{|l|l|l|l|l|l|l|l|l|l|}
\hline HRV/Hormones & $\mathbf{A p - 7}$ & $\mathbf{A p}-\mathbf{6}$ & $\mathbf{A p}-\mathbf{5}$ & $\mathrm{Ap}-4$ & $\mathbf{A p}-\mathbf{3}$ & $\mathbf{A p}-\mathbf{2}$ & $\mathrm{Ap}-1$ & Ap0 & $\mathbf{Z} \pm \mathbf{S E}$ \\
\hline AMo/MxDMn Index & $\mathbf{0 , 3 6}$ & 0,16 & $-0,00$ & $-0,02$ & 0,13 & $-0,04$ & $-0,03$ & 0,08 & $\mathbf{2 , 3 0} \pm \mathbf{0 , 4 8}$ \\
\hline AMo & $\mathbf{0 , 3 2}$ & $\mathbf{0 , 2 2}$ & $-0,05$ & $-0,02$ & 0,12 & $-0,12$ & $-0,07$ & 0,05 & $\mathbf{0 , 3 8} \pm \mathbf{0 , 1 3}$ \\
\hline Triangular Index & $\mathbf{- 0 , 2 3}$ & $\mathbf{- 0 , 2 1}$ & $-0,08$ & $-0,04$ & $-0,16$ & 0,15 & 0,16 & 0,04 & $0,03 \pm 0,19$ \\
\hline MxDMn & $\mathbf{- 0 , 2 3}$ & $\mathbf{- 0 , 1 9}$ & $-0,10$ & $-0,06$ & $-0,19$ & 0,10 & 0,17 & 0,05 & $\mathbf{- 0 , 3 5} \pm \mathbf{0 , 1 2}$ \\
\hline SDNN & $\mathbf{- 0 , 1 9}$ & $-0,17$ & $-0,04$ & $-0,01$ & 0,03 & $\mathbf{0 , 2 0}$ & 0,08 & $-0,09$ & $\mathbf{- 0 , 3 0} \pm \mathbf{0 , 0 9}$ \\
\hline LF band PS & $\mathbf{- 0 , 1 9}$ & $\mathbf{- 0 , 2 0}$ & $-0,03$ & 0,02 & $-0,01$ & $\mathbf{0 , 2 2}$ & 0,14 & $-0,08$ & $\mathbf{0 , 9 1} \pm \mathbf{0 , 3 0}$ \\
\hline HF band PS & $-0,10$ & $-0,14$ & $-0,17$ & $-0,05$ & $-0,13$ & 0,08 & 0,11 & 0,01 & $\mathbf{- 0 , 3 6} \pm \mathbf{0 , 1 7}$ \\
\hline LF/(LF+HF) & $-0,10$ & $-0,05$ & $\mathbf{0 , 2 7}$ & 0,12 & $\mathbf{0 , 2 0}$ & 0,17 & 0,08 & $-0,07$ & $\mathbf{1 , 2 2} \pm \mathbf{0 , 1 0}$ \\
\hline LF/HF & $-0,10$ & $-0,10$ & $\mathbf{0 , 2 4}$ & 0,01 & 0,06 & 0,06 & 0,10 & 0,02 & $\mathbf{1 , 7 1} \pm \mathbf{0 , 3 0}$ \\
\hline 1/Mode & 0,10 & 0,03 & $\mathbf{0 , 2 1}$ & 0,08 & 0,12 & 0,07 & $-0,11$ & $-0,01$ & $\mathbf{- 1 , 2 1} \pm \mathbf{0 , 1 4}$ \\
\hline Baevsky's ARS Index & 0,15 & $-0,04$ & $\mathbf{0 , 2 1}$ & 0,03 & $\mathbf{0 , 1 7}$ & 0,15 & 0,03 & 0,02 & $\mathbf{3 , 3 1} \pm \mathbf{0 , 2 8}$ \\
\hline VLF band PS & $-0,07$ & $-0,05$ & $-0,00$ & $-0,02$ & $\mathbf{0 , 1 6}$ & 0,13 & $-0,01$ & $-0,14$ & $-0,13 \pm 0,44$ \\
\hline ULF band PS & $-0,08$ & $-0,10$ & $-0,03$ & 0,10 & 0,05 & $\mathbf{0 , 3 6}$ & 0,01 & $-0,08$ & $\mathbf{1 , 5 6} \pm \mathbf{0 , 6 9}$ \\
\hline ULF/TP & $-0,10$ & $-0,08$ & $-0,01$ & 0,07 & $-0,01$ & $\mathbf{0 , 1 9}$ & $-0,05$ & $-0,10$ & $\mathbf{0 , 5 5} \pm \mathbf{0 , 2 4}$ \\
\hline Testosterone standard & 0,14 & 0,09 & 0,07 & 0,07 & $-0,01$ & $-\mathbf{0 , 3 0}$ & $-0,13$ & 0,04 & \\
\hline & & & & & & & & $\mathrm{M}$ & $-0,15 \pm 0,20$ \\
\hline & & & & & & & & $\mathrm{F}$ & $\mathbf{1 , 4 3} \pm \mathbf{0 , 4 4}$ \\
\hline Triiodothyronine & $-0,09$ & $-0,15$ & $-0,11$ & $-0,05$ & 0,02 & 0,03 & $-0,15$ & $-\mathbf{0 , 2 7}$ & $-0,34 \pm 0,20$ \\
\hline & & & & & & & & $\mathrm{M}$ & $-0,50 \pm 0,27$ \\
\hline & & & & & & & & F & $-0,16 \pm 0,29$ \\
\hline
\end{tabular}




\begin{tabular}{|l|l|l|l|l|l|l|l|l|l|}
\hline Cortisol & 0,09 & 0,15 & 0,01 & 0,11 & 0,15 & 0,00 & $-0,18$ & $-\mathbf{0 , 2 1}$ & $\mathbf{- 1 , 0 4 \pm 0 , 0 4}$ \\
\hline & & & & & & & & M & $\mathbf{- 1 , 1 2} \pm \mathbf{0 , 0 6}$ \\
\hline & & & & & & & & F & $\mathbf{- 0 , 9 5} \pm \mathbf{0 , 0 6}$ \\
\hline Multiple correlation R & $\mathbf{0 , 4 1 2}$ & $\mathbf{0 , 3 8 1}$ & $\mathbf{0 , 3 3 2}$ & 0,177 & $\mathbf{0 , 3 1 4}$ & $\mathbf{0 , 5 0 6}$ & 0,286 & $\mathbf{0 , 2 9 3}$ & \\
\hline
\end{tabular}

Note. For hormones, Z-scores are given in general, as well as separately for men and women.

According to the canonical correlation analysis, geomagnetic perturbations on the day of EEG registration and for seven days before it determines the HRV\&Endocrine parameters by 59\% (Table 11 and Fig. 7).

\section{Table 11. Factor Structure Matrix for Canonical Roots of Ap-Indices and HRV\&Endocrine parameters}

\begin{tabular}{|c|c|}
\hline Left set & $\mathbf{R}$ \\
\hline Ap-2, nT & 0,641 \\
\hline Ap-5, nT & 0,203 \\
\hline Ap-3, nT & 0,165 \\
\hline Ap-7, nT & $-0,151$ \\
\hline Ap-6, nT & $-0,025$ \\
\hline Ap-0, nT & $-0,104$ \\
\hline Right set & $\mathbf{R}$ \\
\hline 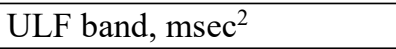 & 0,429 \\
\hline ULF/TP, \% & 0,311 \\
\hline VLF band, msec $^{2}$ & 0,257 \\
\hline Baevsky's ARSI, units & 0,197 \\
\hline $\mathrm{LF} /(\mathrm{LF}+\mathrm{HF}), \%$ & 0,161 \\
\hline $\mathrm{LF} / \mathrm{HF}$ & 0,150 \\
\hline 1/Mode, $\mathrm{msec}^{-1}$ & 0,090 \\
\hline Testosterone, Z-score & $-0,789$ \\
\hline AMo, $\%$ & $-0,146$ \\
\hline Vegetative Balance Ind, un & $-0,076$ \\
\hline SDNN, msec & 0,258 \\
\hline Triangular Index, units & 0,139 \\
\hline MxDMn, msec & 0,089 \\
\hline LF band, $\mathrm{msec}^{2}$ & 0,086 \\
\hline Triiodothyronine, $\mathrm{nM} / \mathrm{L}$ & 0,336 \\
\hline Cortisol, nM/L & 0,200 \\
\hline
\end{tabular}

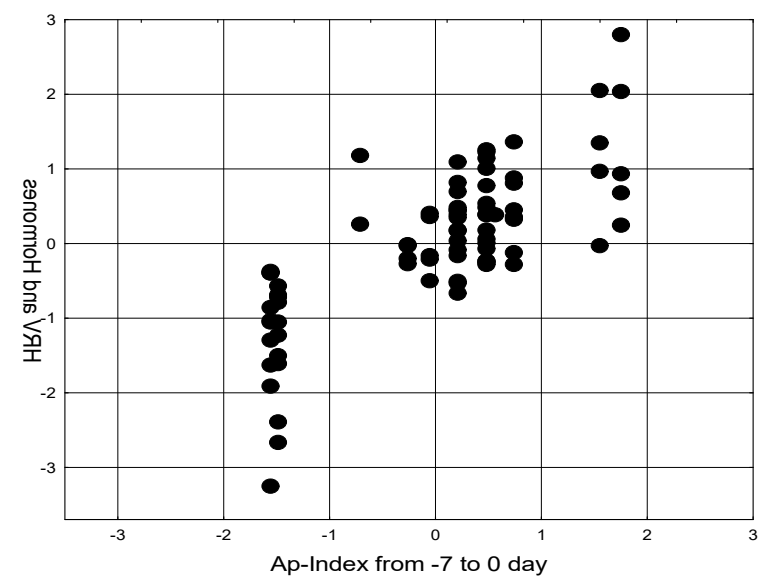

$\mathrm{R}=0,766 ; \mathrm{R}^{2}=0,589 ; \chi^{2}(120)=194 ; \mathrm{p}<10^{-4} ; \Lambda$ Prime $=0,060$

Fig. 7. Scatterplot of canonical correlation between Ap Geomagnetic indices before 7 days and on day testing (X-line) and HRV\&Hormonal parameters (Y-line)

To create a holistic picture of the impact of geomagnetic perturbations on the inseparable triune neuroendocrine-immune complex, we consider it necessary to return to the parameters of immunity [21]. 
Maximum coefficients of multiple correlation with immunity parameters were detected for Ap-index on the eve of blood sampling and 5 days before it while the minimum on 3 and 2 days before sampling (Table 12). The immunity parameters, as a result of canonical correlation analysis, were retrospectively distributed in the correlation matrix between two pairs of canonical roots.

In particular, on the eve of blood sampling, i.e. at the peak of Ap/Immune correlations, there is a maximum suppressive (downregulating) effect of the disturbances of the geomagnetic field on phagocytosis, as well as T-killer and B-lymphocytes, CIC and Igg M\&A levels while upregulating effect on NK-lymphocytes level.

Instead, on the day of blood sampling, the connections with phagocytose, T-killer and NK-lymphocytes, CIC and IgM significantly weaken or disappear, with B-lymphocytes do not change, however, with IgA are strengthened, and with T-helper lymphocytes become significant.

On the other hand, 2 days before testing, there was no significant correlation of these parameters with Apindex, however, there were positive connections with pan-lymphocytes and negative - with monocytes and Creactive protein level.

It seems that the disturbances of the geomagnetic field downregulates with $1 \div 0,5$ day delay the activity, intensity and, especially, the completion of phagocytosis by neutrophils of both gram-positive and gram-negative microbes, and the level in the blood of B-lymphocytes and their secretion of Igg M and A (but not IgG) with the formation of CIC as well as the level of T-helper and T-killer lymphocytes, instead upregulates the level of NKlymphocytes. The downregulation the level of monocytes and C-RP (but not IL-1 $\beta$ ), as well as the upregulation the level of pan-lymphocytes is realized with a delay of $2 \div 1,5$ days.

The second peak of Ap/Immune correlations was detected 5 days before testing. But, despite the almost identical values of $\mathrm{R}, \mathrm{R}^{2}$ and Adjusted $\mathrm{R}^{2}$ with the first peak, their structure differs significantly.

In particular, negative connections of Ap-5 (as well as Ap-6 and Ap-7) with phagocytosis parameters as well as CIC and IgM (but not IgA and Th) are reversed to positive or leveled. In addition, there are significant links with levels of eosinophils as well as T-active and T-regulatory lymphocytes.

\section{Table 12. Matrix of correlations between Ap-Indices and Immunity parameters}

\begin{tabular}{|c|c|c|c|c|c|c|c|c|c|}
\hline Variables for first pair & Ap-7 & Ap-6 & Ap-5 & Ap-4 & Ap-3 & Ap-2 & Ap-1 & Ap0 & $\mathbf{Z} \pm \mathbf{S E}$ \\
\hline Killing Index vs St. aur., \% & 0,16 & $-0,08$ & 0,43 & 0,42 & 0,36 & 0,17 & $-0,55$ & $-0,39$ & $-1,84 \pm 0,13$ \\
\hline Killing Index vs E. coli, \% & 0,37 & 0,21 & 49 & 0,43 &, 42 & 0,14 & $-0,51$ & $-0,18$ & $-2,54 \pm 0,09$ \\
\hline BCC vs E. coli, $10^{9} \mathrm{Bac} / \mathrm{L}$ & 0,28 & & & & 32 & & & & \\
\hline BCC vs St. aur., $10^{9} \mathrm{Bac} / \mathrm{L}$ & 0,24 & 0,21 & 0,28 & 0,33 & 0,35 & 0,02 & $-0,48$ & $-0,21$ & $-1,45 \pm 0,34$ \\
\hline Microb Count E. coli, B/Ph & 0,30 & $-0,14$ & $-0,11$ & &, 21 & 0,02 & $-0,44$ & $-0,41$ & \\
\hline Microb Count St. aur., B/Ph & 0,48 & 0,41 & 0,09 & 0,25 & 0,31 & 0,08 & $-0,35$ & $-0,01$ & $-0,21 \pm 0,10$ \\
\hline Immunoglobulins $\mathrm{M}, \mathrm{g} / \mathrm{L}$ & 0,12 & 0,24 & 0,14 & &, 09 & $-0,01$ & $-0,24$ & $-0,09$ & \\
\hline $\mathrm{CD}_{22}{ }^{+}$B-Lymphocytes, $\%$ & $-0,08$ & $-0,17$ & $-0,02$ & 0,03 & $-0,06$ & $-0,11$ & $-0,20$ & $-0,19$ &, 19 \\
\hline pl, un & $-0,02$ & 0,02 & 0,19 & 0,13 & 0,07 & $-0,01$ & $-0,21$ & $-0,08$ & \\
\hline $\mathrm{CD} 3$ & 0,00 & 0,07 & 0,13 & 0,09 & 0,06 & 0,00 & $-0,21$ & $-0,05$ & \\
\hline hocytes, \% & 0,12 & 0,21 & 0,05 & $-0,06$ & 0,06 & 0,09 & 0,26 & 0,26 & \\
\hline tes, $\%$ & 0,07 & 0,07 & $-0,01$ & & $-0,02$ & & & & \\
\hline pair & Ap-7 & Ap-6 & Ap-5 & Ap-4 & Ap-3 & Ap-2 & Ap-1 & Ap0 & \\
\hline & & & & & & & & & \\
\hline $\mathrm{CD3}$ & $-0,14$ & $-0,26$ & $-0,19$ & 0 , & $-0,05$ & 0,01 & $-0,15$ & $-0,27$ & \\
\hline $\mathrm{CD}$ & & & & & & & & & \\
\hline vs E.coli, \% & $-0,04$ & 0,07 & $-0,28$ & $-0,03$ & $-0,10$ & $-0,21$ & $-0,20$ & $-0,12$ & \\
\hline nocytogram & $-0,03$ & $-0,04$ & $-0,18$ & $-0,07$ & $-0,11$ & $-0,15$ & $-0,12$ & $-0,08$ &, 07 \\
\hline Inter & $-0,07$ & $-0,16$ & $-0,12$ & -0 , & $-0,09$ & $-0,03$ & 0,14 & $-0,03$ & \\
\hline $\mathrm{C}-\mathrm{F}$ & 0,02 & 0,04 & $-0,16$ & -0 , & $-0,04$ & $-0,27$ & 0,06 & 0,03 & \\
\hline Interleuki & 0,02 & 0,05 & $-0,14$ & & $-0,03$ & $-0,23$ & 0,08 & 0,04 & \\
\hline Mon & $-0,13$ & $-0,11$ & $-0,06$ & $-0,12$ & $-0,22$ & $-0,22$ & $-0,01$ & 0,05 & $-0,58$ \\
\hline Lymphocytes il & 0,08 & $-0,06$ & 0,14 & $-0,00$ & 0,13 & 0,23 & 0,17 & 0,02 & \\
\hline ils, $\%$ & 0,02 & 0,08 & 0,20 & & 0,14 & 0,12 & 0,00 & 0,02 & $-2,39 \pm 0,21$ \\
\hline Eos & $-0,08$ & 0,17 & 0,24 & 0,17 & 0,09 & 0,06 & $-0,05$ & 0,08 & $-0,21 \pm 0,22$ \\
\hline ry, $\%$ & 0,10 & 0,24 & 0,27 & 0,06 & 0,09 & $-0,00$ & $-0,08$ & 0,15 & $0,73 \pm 0,15$ \\
\hline Phagocytic Ind vs S & 0,25 & 0,31 & $-0,31$ & $-0,16$ & $-0,06$ & $-0,05$ & 0,25 & $\mathbf{0 , 3 0}$ & \\
\hline Entropy Leukocytogram & $-0,09$ & 0,04 & 0,24 & 0,12 & 0,05 & 0,08 & 0,03 & 0,08 & $-1,16 \pm 0,12$ \\
\hline PMN Neutropl & $-0,01$ & 0,03 & $-0,16$ & 0,00 & $-0,04$ & $-0,12$ & $-0,15$ & $-0,09$ & $0,78 \pm 0,12$ \\
\hline Leukocytes in total, $10^{9} / \mathrm{L}$ & $-0,06$ & 0,19 & 0,04 & $-0,02$ & 0,04 & $-0,15$ & $-0,04$ & 0,07 & $1,20 \pm 0,26$ \\
\hline Immunoglobulins $\mathrm{G}, \mathrm{g} / \mathrm{L}$ & 0,07 & 0,02 & 0,07 & 0,07 & 0,09 & $-0,03$ & $-0,14$ & $-0,07$ & $1,71 \pm 0,15$ \\
\hline
\end{tabular}




\begin{tabular}{|l|l|l|l|l|l|l|l|l|l|}
\hline Multiple correlation R & $\mathbf{0 , 6 6 2}$ & $\mathbf{0 , 7 0 7}$ & $\mathbf{0 , 7 5 8}$ & $\mathbf{0 , 5 9 5}$ & $\mathbf{0 , 5 4 1}$ & $\mathbf{0 , 4 7 9}$ & $\mathbf{0 , 7 6 8}$ & $\mathbf{0 , 6 0 8}$ & \\
\hline
\end{tabular}

Finally, the relationship between Ap-indices for $7 \div 0$ days, on the one hand, and the registered immunity parameters, on the other hand, was analyzed. The analysis revealed two significant pairs of canonical roots.

The geomagnetic root of the first pair (Table 13) is expected to receive the maximum positive load from the Ap-index on the eve of blood sampling and the second in rank - from the Ap-index on the day of testing. In contrast, Ap-7, Ap-4 and Ap-3 indices give negative factor loads.

The immune root of the first pair is represented mainly by the parameters that are subject to downregulation by variations of the magnetic field with a delay of $1 \div 0,5$ day while to upregulation with a delay of 7, 4 and 3 days. A positive correlation of Ap-1 and Ap0 with the level of 0-lymphocytes also reflects their immunosuppressive effect.

Taken together, the variations of the magnetic field in these periods before blood collection determine this constellation of immune parameters by $85 \%$ (Fig. 8).

\section{Table 13. Factor Structure Matrix for first Ap/Immune pair of Canonical Roots}

\begin{tabular}{|c|c|}
\hline Left set & Root 1 \\
\hline Ap-1, nT & 0,726 \\
\hline Ap- $0, \mathrm{nT}$ & 0,481 \\
\hline Ap-7, nT & $-0,489$ \\
\hline Ap-4, nT & $-0,332$ \\
\hline Ap-3, nT & $-0,290$ \\
\hline Right set & Root 1 \\
\hline Microbial Count for E. coli, Bacteria/Phagocyte & $-0,723$ \\
\hline Bactericidal Capacity vs E. coli, $10^{9} \mathrm{Bac} / \mathrm{L}$ & $-0,557$ \\
\hline Immunoglobulins $\mathrm{A}, \mathrm{g} / \mathrm{L}$ & $-0,459$ \\
\hline Killing Index vs Staph. aureus, \% & $-0,444$ \\
\hline Microbial Count for Staph. aureus, Bacter/Phag & $-0,438$ \\
\hline Bactericidal Capacity vs Staph. aureus, $10^{9} \mathrm{Bac} / \mathrm{L}$ & $-0,433$ \\
\hline Killing Index vs E. coli, \% & $-0,374$ \\
\hline Phagocytosis Index vs E. coli, \% & $-0,344$ \\
\hline Immunoglobulins $\mathrm{M}, \mathrm{g} / \mathrm{L}$ & $-0,342$ \\
\hline $\mathrm{CD}^{+}{ }^{+} \mathrm{CD} 4^{+}$T-helper Lymphocytes & $-0,302$ \\
\hline Entropy of Immunocytogram & $-0,244$ \\
\hline CD22 ${ }^{+}$B-Lymphocytes, $\%$ & $-0,241$ \\
\hline $\mathrm{CD}^{+}$T-active Lymphocytes, $\%$ & $-0,121$ \\
\hline Circulating Immune Complex, units & $-0,110$ \\
\hline $\mathrm{CD}^{+} \mathrm{CD} 8^{+} \mathrm{T}$-cytolytic, $\%$ & $-0,110$ \\
\hline 0-Lymphocytes, $\%$ & 0,322 \\
\hline $\mathrm{CD}^{2} 6^{+}$NK-Lymphocytes, $\%$ & 0,122 \\
\hline
\end{tabular}




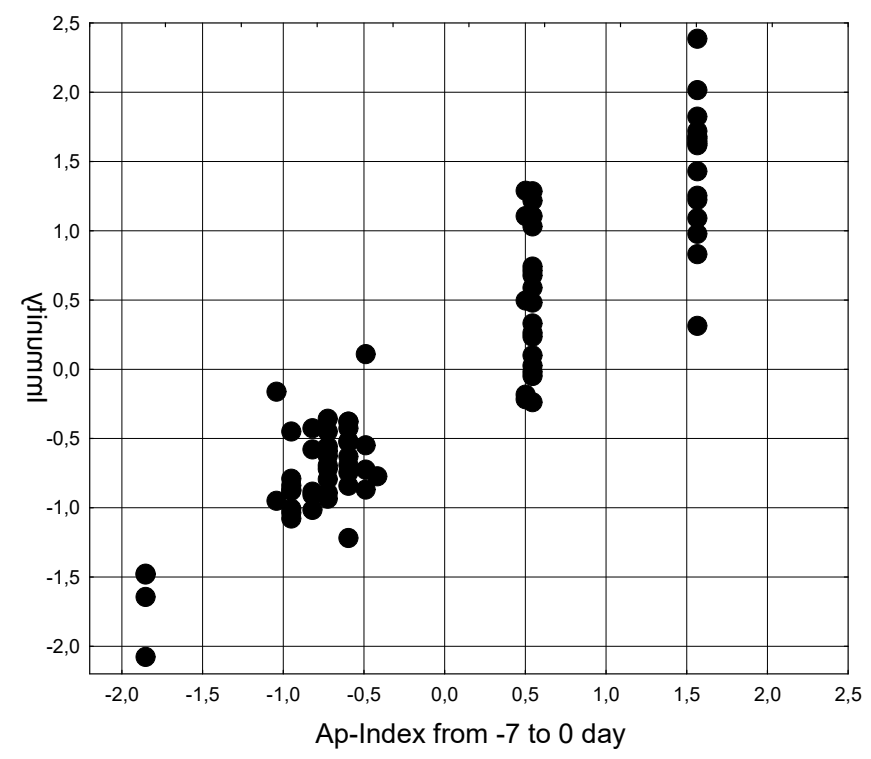

$\mathrm{R}=0,921 ; \mathrm{R}^{2}=0,849 ; \chi^{2}(200)=375 ; \mathrm{p}<10^{-6} ; \Lambda$ Prime $=0,0028$

Fig. 8. Scatterplot of canonical correlation between Ap Geomagnetic indices before 7 days and on day blood sampling (X-line) and Immune parameters (Y-line). First pair of Roots

The geomagnetic root of the second pair was without the top component of the first pair (Ap-1), and all seven components give a load with the same sign (Table 14).

The immune root of the second pair is represented by rod-shaped neutrophils and eosinophils which are subject to the upregulation by Ap-4, by monocytes, IL-6 and C-reactive protein downregulated by Ap-2 and Lymphocytes in total upregulated by Ap-2. But the main array is represented by immune parameters that are subject to suppression or activation by the variations of the magnetic field on other days.

Table 14. Factor Structure Matrix for second Ap/Immune pair of Canonical Roots

\begin{tabular}{|l|l|}
\hline Left set & Root 2 \\
\hline Ap-6, nT & $\mathbf{0 , 7 4 5}$ \\
\hline Ap-7, nT & $\mathbf{0 , 6 5 4}$ \\
\hline Ap-3, nT & $\mathbf{0 , 4 8 3}$ \\
\hline Ap-5, nT & $\mathbf{0 , 4 4 0}$ \\
\hline Ap-0, nT & $\mathbf{0 , 3 2 8}$ \\
\hline Ap-2, nT & $\mathbf{0 , 2 2 7}$ \\
\hline Ap-4, nT & $\mathbf{0 , 3 1 1}$ \\
\hline Right set & $\mathbf{R o o t} \mathbf{2}$ \\
\hline Immunoglobulins A, g/L & $\mathbf{- 0 , 6 0 3}$ \\
\hline CD3 $^{+}$CD4 ${ }^{+}$T-helper Lymphocytes, \% & $\mathbf{- 0 , 4 8 4}$ \\
\hline CD3 $^{+}$T-active Lymphocytes, \% & $\mathbf{- 0 , 2 8 9}$ \\
\hline CD22 ${ }^{+}$B-Lymphocytes, \% & $\mathbf{- 0 , 2 5 5}$ \\
\hline Phagocytosis Index vs E. coli, \% & $\mathbf{- 0 , 2 4 2}$ \\
\hline Entropy of Immunocytogram & $\mathbf{- 0 , 2 0 8}$ \\
\hline Interleukin-1, ng/L & $\mathbf{- 0 , 1 5 6}$ \\
\hline Monocytes, \% & $\mathbf{- 0 , 2 2 6}$ \\
\hline C-Reactive Protein, mg/L & $\mathbf{- 0 , 0 5 8}$ \\
\hline Interleukin-6, ng/L & $\mathbf{- 0 , 0 5 7}$ \\
\hline Lymphocytes in total, \% & $\mathbf{0 , 2 1 6}$ \\
\hline Microbial Count for Staph. aureus, Bac/Phagocyte & $\mathbf{0 , 5 2 0}$ \\
\hline Killing Index vs E. coli, \% & $\mathbf{0 , 5 1 5}$ \\
\hline CD4 ${ }^{+}$CD25 ${ }^{+}$T-regulatory Lymphocytes, \% & $\mathbf{0 , 3 8 1}$ \\
\hline 0-Lymphocytes, \% & $\mathbf{0 , 3 5 7}$ \\
\hline Phagocytosis Index vs Staph. aureus, \% & $\mathbf{0 , 2 7 0}$ \\
\hline
\end{tabular}




\begin{tabular}{|l|l|}
\hline Bactericidal Capacity vs Staph. aureus, $10^{9} \mathrm{Bac} / \mathrm{L}$ & $\mathbf{0 , 2 6 2}$ \\
\hline Bactericidal Capacity vs E. coli, $10^{9}$ Bacteria/L & $\mathbf{0 , 1 7 2}$ \\
\hline Rod-shaped Neutrophils, \% & $\mathbf{0 , 1 8 0}$ \\
\hline Eosinophils, $\%$ & $\mathbf{0 , 1 4 8}$ \\
\hline
\end{tabular}

Taken together, the variations of the magnetic field determine this constellation of immune parameters by $74 \%$ (Fig. 9).

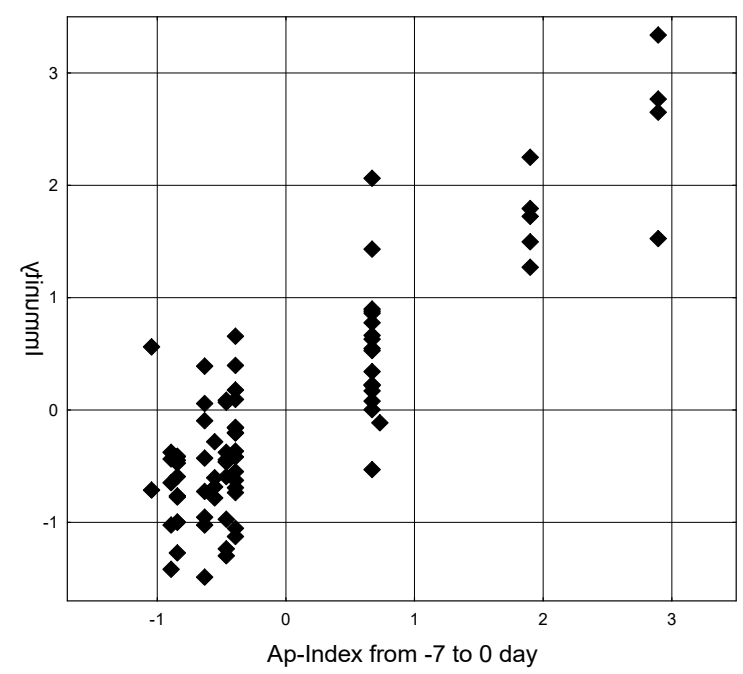

$\mathrm{R}=0,862 ; \mathrm{R}^{2}=0,743 ; \chi^{2}(168)=255 ; \mathrm{p}<10^{-4} ; \Lambda$ Prime $=0,0187$

Fig. 9. Scatterplot of canonical correlation between Ap Geomagnetic indices before 7 days and on day blood sampling (X-line) and Immune parameters (Y-line). Second pair of Roots

Collected patterns of coefficients of multiple correlation show the best congruence for the parameters of immunity and EEG, especially in the range from -5 to 0 days for testing (Fig. 10).

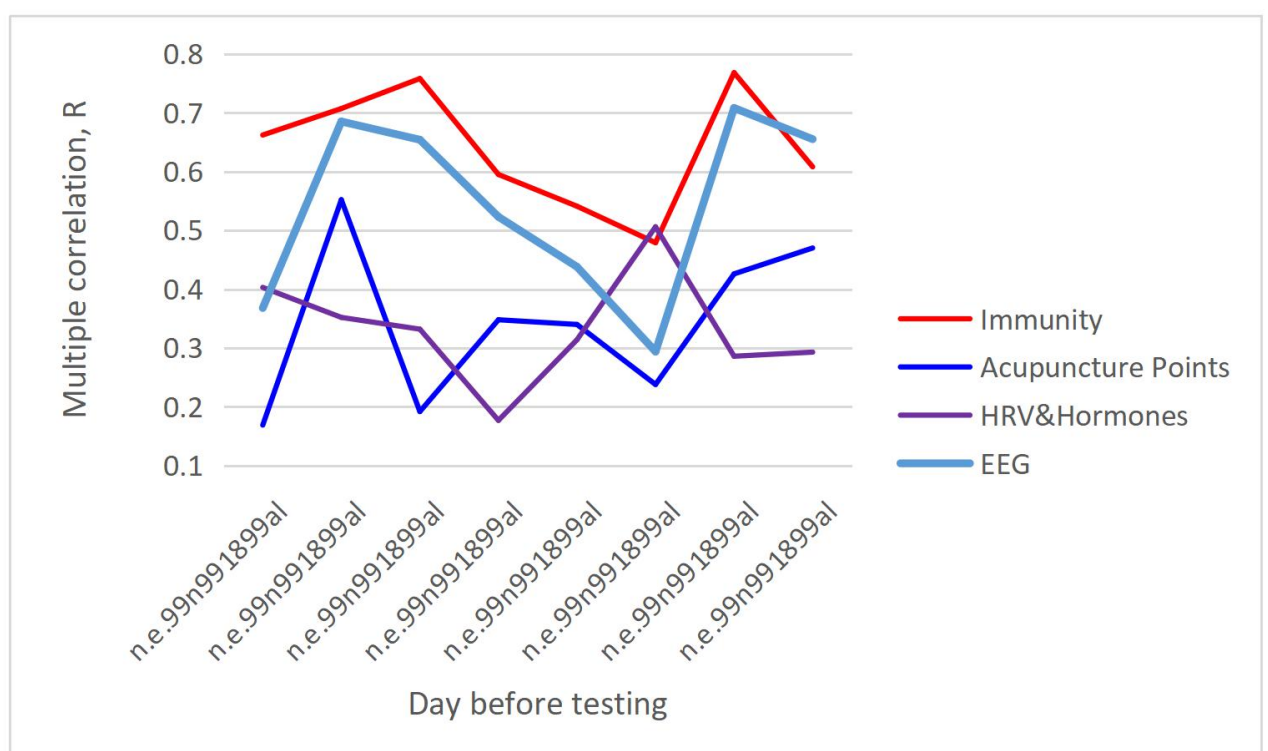

Fig. 10. Patterns of coefficients of multiple correlation $R$ between Ap-Indices and acupuncture points as well as neuro-endocrine-immune complex parameters

The visual impression is confirmed by the results of canonical correlation analysis, as a result of which two pairs of roots were formed.

The EEG Root of the first pair (Table 15) contains information about the PSD loci of the scalp, on which the nerve nuclei that generate delta, theta and beta rhythms are projected, which mainly have a suppressive effect on phagocytosis parameters, blood levels of B-lymphocytes and their secreted IgM as well as T-regulatory lymphocytes, which are present in the Immune Root. The opposite sign factor load on the level of 0-lymphocytes 
additionally reflects a decrease in the expression of at least CD22, CD4 and CD25 receptors. Naturally, this immunotropic effect is accompanied by a decrease in the Entropy of the Immunocytogram.

\section{Table 15. Factor Structure Matrix for first pair of Canonical Roots of EEG and Immune parameters}

\begin{tabular}{|c|c|}
\hline Left set & R1 \\
\hline PSD T6- $\delta, \mu V^{2} / H z$ & $-0,372$ \\
\hline PSD P4- $\beta, \%$ & $-0,335$ \\
\hline PSD T6- $\theta, \mu V^{2} / H z$ & $-0,286$ \\
\hline PSD T3- $\delta, \%$ & $-0,279$ \\
\hline PSD F8- $\delta, \mu V^{2} / H z$ & $-0,269$ \\
\hline PSD F7- $\delta, \%$ & $-0,220$ \\
\hline PSD F7- $\beta, \mu V^{2} / H z$ & $-0,190$ \\
\hline PSD F8- $\theta, \mu V^{2} / H z$ & $-0,165$ \\
\hline PSD 02- $\theta, \%$ & 0,254 \\
\hline PSD T3- $\beta, \%$ & 0,220 \\
\hline PSD F4- $\theta, \%$ & $\mathbf{0 , 1 5 0}$ \\
\hline Right set & R1 \\
\hline Entropy of Immunocytogram & 0,549 \\
\hline CD22 ${ }^{+}$B-Lymphocytes, $\%$ & 0,532 \\
\hline Killing Index vs E. coli, \% & 0,329 \\
\hline Microbial Count for Staph. aureus, Bacter/Phag & 0,303 \\
\hline Immunoglobulins $\mathrm{M}, \mathrm{g} / \mathrm{L}$ & 0,269 \\
\hline $\mathrm{CD}^{+}{ }^{+} \mathrm{CD} 25^{+}$T-regulatory Lymphocytes, $\%$ & 0,247 \\
\hline Phagocytosis Index vs Staph. aureus, \% & 0,181 \\
\hline Bactericidal Capacity vs Staph. aureus, $10^{9} \mathrm{~B} / \mathrm{L}$ & 0,137 \\
\hline 0-Lymphocytes, \% & $-0,524$ \\
\hline Phagocytosis Index vs E. coli, $\%$ & $-0,441$ \\
\hline
\end{tabular}

The degree of neurogenic immunomodulation is very significant: $89 \%$ (Fig. 11).

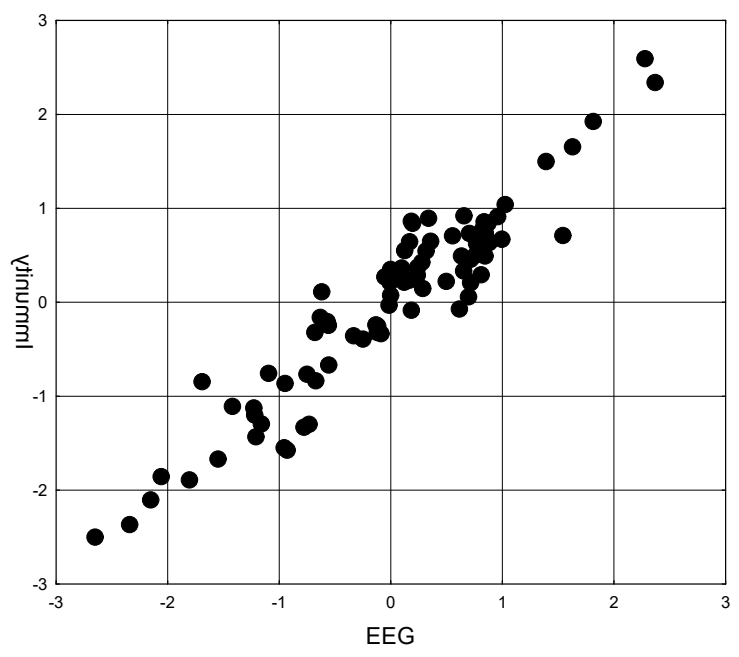

$\mathrm{R}=0,944 ; \mathrm{R}^{2}=0,892 ; \chi^{2}(624)=794 ; \mathrm{p}<10^{-5} ; \Lambda$ Prime $<10^{-6}$

Fig. 11. Scatterplot of canonical correlation between EEG (X-line) and Immunity (Y-line) parameters. First pair of Roots

The EEG Root of the second pair (Table 16) is represented by another constellation of neural structures that mainly enhance serum Igg A and M levels, regulatory T lymphocytes level as well as E. coli phagocytosis parameters. 
Table 16. Factor Structure Matrix for second pair of Canonical Roots of EEG and Immune parameters

\begin{tabular}{|c|c|}
\hline Left set & $\mathbf{R 2}$ \\
\hline PSD C $4-\theta, \%$ & 0,247 \\
\hline PSD T3- $\theta, \%$ & 0,247 \\
\hline PSD F3- $\beta, \%$ & 0,247 \\
\hline PSD F7- $\beta, \%$ & 0,239 \\
\hline PSD Fp1- $\theta, \%$ & 0,234 \\
\hline PSD C3- $\theta, \%$ & 0,225 \\
\hline PSD T6- $\beta, \%$ & 0,224 \\
\hline PSD F7- $\theta, \%$ & 0,220 \\
\hline PSD T6- $\theta, \%$ & 0,216 \\
\hline PSD T4- $\beta, \%$ & 0,215 \\
\hline PSD F8- $\theta, \%$ & 0,196 \\
\hline PSD F4- $\theta, \%$ & 0,185 \\
\hline PSD T6- $\delta, \mu V^{2} / H z$ & 0,145 \\
\hline PSD T3- $\beta, \%$ & 0,127 \\
\hline PSD T5- $\delta, \%$ & $-0,221$ \\
\hline PSD F7- $\delta, \%$ & $-0,203$ \\
\hline PSD T3- $\delta, \%$ & $-0,163$ \\
\hline Right set & $\mathbf{R 2}$ \\
\hline Immunoglobulins $\mathrm{A}, \mathrm{g} / \mathrm{L}$ & 0,383 \\
\hline Killing Index vs E. coli, \% & 0,340 \\
\hline $\mathrm{CD}^{+}{ }^{+} \mathrm{CD} 25^{+}$T-regulatory Lymphocytes, $\%$ & 0,224 \\
\hline Bactericidal Capacity vs E. coli, $10^{9}$ Bacteria/L & 0,191 \\
\hline Immunoglobulins $\mathrm{M}, \mathrm{g} / \mathrm{L}$ & 0,185 \\
\hline
\end{tabular}

The degree of neurogenic immunomodulation of this immune constellation is smaller, but still quite significant: $82 \%$ (Fig. 12).

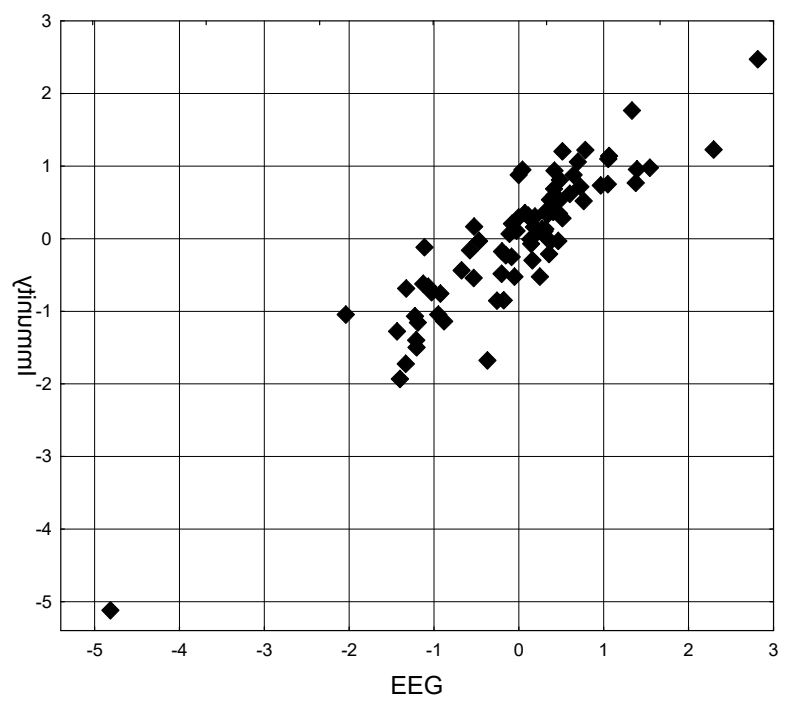

$\mathrm{R}=0,908 ; \mathrm{R}^{2}=0,824 ; \chi^{2}(570)=676 ; \mathrm{p}=0,002 ; \Lambda$ Prime $<10^{-5}$

Fig. 12. Scatterplot of canonical correlation between EEG (X-line) and Immunity (Y-line) parameters. Second pair of Roots

In contrast, the degree of immunomodulation induced by circulating catecholamines, sympathetic and vagus nerves, and adaptation hormones was found to be weaker than neurogenic: $51 \%$ (Table 17 and Fig. 13). 
Table 17. Factor Structure Matrix for Canonical Roots of HRV\&Hormonal and Immune parameters

\begin{tabular}{|l|l|}
\hline Left set & R1 \\
\hline 1/Mode as Circulating Cathecholamines, msec $^{-1}$ & $\mathbf{0 , 7 6 6}$ \\
\hline Testosterone standardized by sex\&age, Z-score & $\mathbf{0 , 4 2 6}$ \\
\hline AMo/MxDMn as Vegetative Balance Index, un & $\mathbf{0 , 2 5 5}$ \\
\hline Amplitude of Mode as Sympathetic tone, \% & $\mathbf{0 , 1 9 9}$ \\
\hline Cortisol, nM/L & $\mathbf{0 , 0 5 9}$ \\
\hline ULF/TP & $\mathbf{- 0 , 3 1 9}$ \\
\hline MxDMn as Vagal tone, msec & $\mathbf{- 0 , 2 8 1}$ \\
\hline Triangular Index as Vagal tone, units & $\mathbf{- 0 , 2 7 6}$ \\
\hline ULF band, msec ${ }^{2}$ & $\mathbf{- 0 , 2 0 1}$ \\
\hline SDNN as Vagal tone, msec & $\mathbf{- 0 , 1 6 6}$ \\
\hline Triiodothyronine, nM/L & $\mathbf{- 0 , 1 2 2}$ \\
\hline Testosterone actual, nM/L & $\mathbf{- 0 , 0 9 5}$ \\
\hline Right set & $\mathbf{R 1}$ \\
\hline Killing Index vs Staph. aureus, \% & $\mathbf{0 , 5 2 7}$ \\
\hline Microbial Count for E. coli, Bacteria/Phagocyte & $\mathbf{0 , 4 0 3}$ \\
\hline Phagocytosis Index vs E. coli, \% & $\mathbf{0 , 3 7 5}$ \\
\hline Bactericidal Capacity vs Staph. aureus, 10 ${ }^{9}$ B/L & $\mathbf{0 , 3 7 2}$ \\
\hline Bactericidal Capacity vs E. coli, $10^{9}$ Bacteria/L & $\mathbf{0 , 1 7 2}$ \\
\hline Phagocytosis Index vs Staph. aureus, \% & $\mathbf{0 , 1 4 8}$ \\
\hline 0-Lymphocytes, \% & $\mathbf{0 , 3 2 3}$ \\
\hline CD22 ${ }^{+}$B-Lymphocytes, \% & $\mathbf{- 0 , 2 3 6}$ \\
\hline CD3 ${ }^{+}$CD4 ${ }^{+}$T-helper Lymphocytes & $\mathbf{- 0 , 2 1 7}$ \\
\hline Killing Index vs E. coli, \% & $\mathbf{- 0 , 2 0 6}$ \\
\hline
\end{tabular}

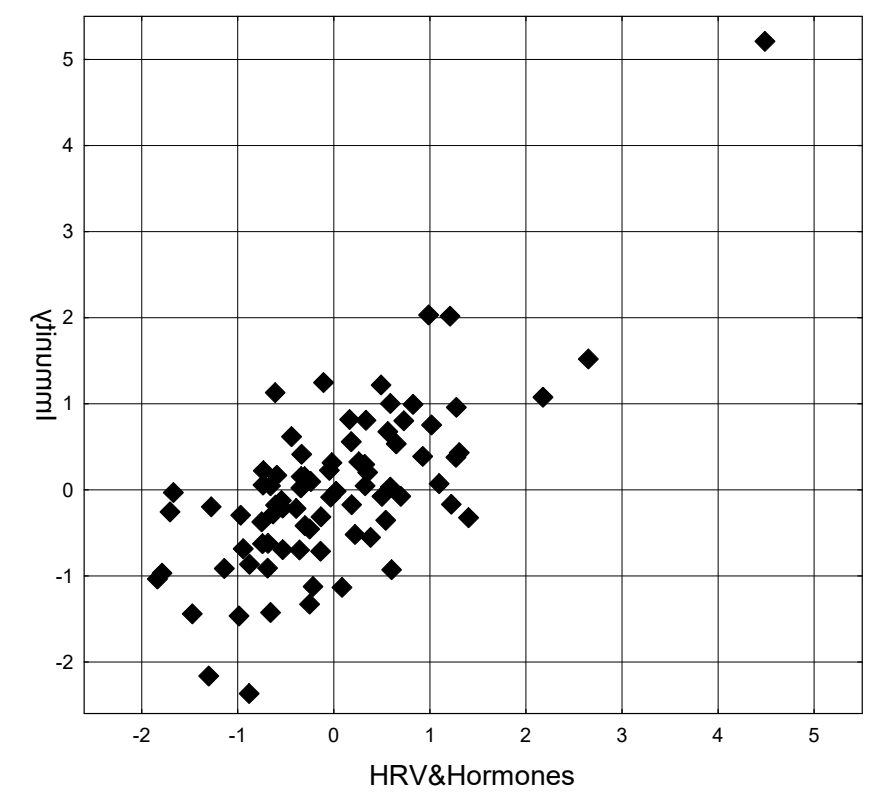

$\mathrm{R}=0,714 ; \mathrm{R}^{2}=0,510 ; \chi^{2}(140)=164 ; \mathrm{p}=0,049 ; \Lambda$ Prime $=0,090$

Fig. 13. Scatterplot of canonical correlation between HRV\&Hormonal (X-line) and Immunity (Yline) parameters

DISCUSSION 
The presented results fit into the concept that Solar-induced fluctuations in the ambient geomagnetic field have been correlated with a wide range of biological effects [22Muehsam D and Ventura C, 2014], including changes in EEG [23Babayev ES and Allahverdiyeva AA, 2007; 24Mulligan BP et al, 2010; 25/Novik OB, Smirnov FA.] and HRV [26Baevsky RM et al;27 McCraty] parameters in humans. Let's dwell in more detail on the research closest to ours in design. By the way, our study was not planned in advance, it is the result of a retrospective analysis of databases of previous projects, but carried out by the same performers on the same equipment. So, in essence, this is a truly double-blind study.

Our former compatriots Babayev and Allahverdiyeva [23/2007], employing, like us, quantitative electroencephalographic (QEEG) technology, carried out investigations involving 27 functionally healthy females (permanent group), aged 20-40 years old. The EEG measurements (in 16 loci) were performed every day for each person at the same time of the day during geomagnetic quiet days. In the days with different levels of disturbances of geomagnetic conditions (few moderate and major geomagnetic storms: March 31, 2001; November 8-10, 2004; January 17-19, May 17, June 15, August 24, September 11-12, 2005; as well as some severe/extreme storms: July 2000; October-November 2003) the experiments were carried out several times.

Unfortunately, despite the declared quantitative approach to EEG analysis, the authors give only a qualitative description of the changes. The authors demonstrated conspicuous and specific changes in alpha and theta activity within the right hemispheres of normal subjects during major geomagnetic storms. There were almost no significant complaints about functional state in periods of weakly-disturbed geomagnetic conditions. Weak and severe geomagnetic storms affect the functional state of the human brain in a different way. It is established that weak and moderate geomagnetic storms exert stimulating influence while the strong disturbances of the geomagnetic conditions activate braking (inhibiting) processes. In the days of weak geomagnetic disturbances, no significant changes in the human brain activity were observed. Some negligible shifts, registered in several examinees, reflected an increased activity of meso-diencephalic structures usually observed during the activation of an organism. In comparison with the above-mentioned results, in the days with severe geomagnetic storms the human brain's activity is seriously disintegrated. The normal functioning of integrative non-specific systems, located within the limits of limbric-reticular complex and responsible for creation of the relevant level of wakefulness, which is directed on realization of optimum current activity of an organism, is broken. The imbalance of activating and deactivating mechanisms also includes dysfunctions of ergo- and trophotropic over-segmentary centers. Babayev and Allahverdiyeva [23/2007] concluded that changes in geomagnetic conditions mostly affect the activity of regulating systems, which are related to high cortical mechanisms of regulation and subcortical integrative apparatuses responsible for organization of routine activity of an organism, and for adaptation to changes of a physical environment.

For our part, we want to emphasize that theta and alpha rhythms were sensitive to geomagnetic disturbances, as in our study.

Previously Persinger and Richards [28/1995] report here similar QEEG correlations, primarily involving the right hemisphere, during the much less intense periods of recent geomagnetic activity during the hiatus between Solar Cycle 23 and 24 in samples of healthy young adults. The magnitude of global (and local) geomagnetic variations was within the range $(15-20 \mathrm{nT})$ correlated with enhanced vestibular experiences during partial sensory deprivation in healthy adults. Impressed, in our opinion, by the results of their Azerbaijani colleagues, and so strongly that they included the name of the country in the title of their article, the Canadians repeated the research of Azerbaijanis.

In Experiments I, Mulligan, Hunter and Persinger [24] analyzed the correlation between Kp-indices for the interval between 1 July 2006 - 31 January 2008 (geomagnetic variations was within the range 20-25 nT) and QEEG parameters in healthy young people of both sexes. To discern the strength of effect a functional canonical correlation was completed between the beta power within the left parietal $(\mathrm{P} 3)$ region $(\mathrm{r}=-0,66)$ and the gamma power within the right frontal $(\mathrm{F} 8)$ region $(\mathrm{r}=-0,71)$ as dependent variables and the Kp value during the interval of the recording as the covariate. The equivalent multiple $\mathrm{R}$ was 0,73 .

In our observation, the correlation of Ap/PSD P3- $\beta$ was 0,10 and 0,02 on the day of registration, and on the eve of it 0,09 and $-0,03$ to relative and absolute PSD, respectively. We were not able to record the gamma rhythm, instead we recorded the EEG at 16 loci against 8 loci (F7, F8, T3, T4, P3, P4. O1, O2) in the cited study. And just in the C3 locus relative PSD- $\beta$ was positively correlated with Ap-index on the day of registration $(r=0,30)$ and on the eve of it $(r=0,24)$, while with the $C 4$ locus the connections were weaker $(0,21$ and 0,19$)$.

Instead, our data are in principle consistent with the cited study on the inhibitory effect of geomagnetic field disturbances on PSD theta rhythm. The authors used an unconventional approach when calculating correlations between power values within each of the $1 \mathrm{~Hz}$ intervals and $\mathrm{Kp}$ values. Partial correlational analyses revealed the strongest correlation $(\mathrm{r}=-0,58)$ was between $\mathrm{Kp}$ values and power within the 8-8,9 $\mathrm{Hz}$ over the right [lateral] frontal (F8) lobe as well as the power within the 7-7,9 Hz band over the left parietal (P3) region ( $\mathrm{r}=-$ $0,57)$. The equivalent multiple $\mathrm{R}$ was 0,66 . In our study, the PSD of total theta band $(4-7 \mathrm{~Hz})$ connections for individual loci are much weaker, while the locus area is much larger, so that the canonical correlation between 
theta rhythm and Ap-index was almost similar (see Table 8). In addition, we found a correlation of Ap/PSD T6alpha $(\mathrm{r}=-0,25)$.

In Experiments II by Mulligan et al, the results of which were published in the same paper [24], the correlation between QEEG parameters and Ap-indices for the interval between 9-20 March 2008 (geomagnetic variations was within the range 6-30 nT). For the geomagnetic correlations (Ap-index), the strongest and most significant correlations for the wide-band were for gamma in the right [anterior] temporal (T4) lobe and theta in the right parietal (P4) lobe, similar to our data. For the successive $1-\mathrm{Hz}$ bands within the theta range, geomagnetic activity was correlated negatively with the 7-7,9 $\mathrm{Hz}$ band in the right frontal (F8) lobe while both the $3-3,9 \mathrm{~Hz}$ and $5-5,9 \mathrm{~Hz}$ bands were positively correlated within the right parietal (P4) lobe.

For the following correlation analysis, the authors for some reason used atmospheric power instead of Ap-index (correlation between them, however, is 0,93). As atmospheric power increased during the recordings power within the theta band decreased within the right frontal $(\mathrm{F} 8)$ region $(\mathrm{r}=-0,81)$ while there was general activation in the beta range over the right [anterior] temporal (T4) lobe $(\mathrm{r}=0,38)$ and in the gamma range for the temporal lobes of right $(\mathrm{r}=0,36)$ and left $(\mathrm{r}=0,36)$ hemispheres. Interestingly, that negative correlations for the 1$\mathrm{Hz}$ increments within the $\mathrm{F} 8$ theta $(6-6,9 \mathrm{~Hz})$ and $((7-7,9 \mathrm{~Hz})$ are inverted into positive ones within the F8 (5-5,9 Hz), F7 (4-4,9 Hz), F7 (8-8,9 Hz) and P3 (4-4,9 Hz).

The Schumann-absorption model proposed by Cherry (29/2002) and others presumes that maximal energy transfer between weak magnetic variations within the geomagnetic-ionospheric system (30/Schlegel and Fuellekrug, 1999) occur when the exogenous frequencies correspond with those measured from the brain by EEG. The peak absorption would involve the theta $(4-8 \mathrm{~Hz})$ and alpha $(8-13 \mathrm{~Hz})$ bands, which include the base and first harmonic of the Schumann resonance (31/Konig et al, 1981). Mulligan et al [24/2010] reasoned that energy transfer could be inferred by more or less "instantaneous" increases and decreases in spectral power within the EEG theta band.

In contrast to the cited authors, we did not find much effect of geomagnetic activity on the right [lateral] frontal (F8) lobe. We also found similarly strong negative correlations for PSD theta rhythm in right parietal (P4) and right [anterior] temporal (T4) while left [lateral] frontal (F7) lobes as well as in both right (C4 and T6) and left ( $\mathrm{C} 3$ and $\mathrm{Fp} 1$ ) loci, which were not involved in the cited studies. In addition, PSD beta rhythm in locus C3 correlates positively with Ap-index. It is believed that the hippocampus is projected at the C3 and C4 loci, and the amygdala at the T3 and T4 loci.

Previously, Persinger and Richards (28/1995) reported that a cluster of experiences loaded on a factor dominated by vestibular sensations significantly increased when global geomagnetic activity exceeded 15-20 nT. The two frequency bands most consistently and strongly correlated with increased geomagnetic activity within the range that occurred during their study were gamma $(35-45 \mathrm{~Hz})$ and theta $(4-7 \mathrm{~Hz})$. Mulligan et al [24/2010] believe that a most parsimonious explanation for an intrinsic connection between these two nonadjacent bands involves a role of the hippocampal formation. These two bands involve the peak energy of the septalhippocampal inputs. In addition, an intrinsic feature of many hippocampal neurons is the superimposition of gamma patterns upon the predominantly 5-7 Hz theta rhythm (32/Buzsaki, 2002). That the hippocampal formation, along with the amygdala, has the lowest threshold for seizure induction is well known (33/Gloor, 1997). The second environmental measure, atmospheric power, revealed similar effects. Again the cerebral region most consistently correlated with this inference of energy between the ionosphere and the Earth's surface was the right frontal lobe. Atmospheric power, measured in Gigawatts, reveals the consequences of the interactions between energetic particulates within the Earth-ionospheric cavity. This is a potentially important ancillary measure for electroencephalographic correlations because magneto-hydrodynamic waves generated within this medium can propagate to the Earth's surface. In both Experiments I and II the correlation between power within the gamma range and either geomagnetic activity or atmospheric power was negative. This means that as the geomagnetic activity or atmospheric power increased the cerebral activity within this band decreased [24/Mulligan et al 2010].

Developing the discussion, Mulligan et al [24/2010] appeal to the position that the right frontal area has powerful functional, primarily inhibitory, connections with the right parietal and temporal regions. Consequently, diminished power or inhibitory frontal functions can encourage disinhibition of functions within the caudal region. The resultant stimulation of activity would produce a sympathetic dominance (arousal), the phenomena that was also shown by Babayev and Allahverdiyeva (2007) during moderate magnetic storms.

It is here that we consider it appropriate to make a small excursion. According to the concept of "central autonomic network (CAN)" (34/Benarroch, 1993; 35/Palma, J. A., and Benarroch, E. E.,2014; 36/Thayer Lane, 2009) it include following cortical, subcortical, and medullary structures: the anterior cingulate, insular, orbitofrontal, and ventromedial cortices; the central nucleus of the amygdala; the paraventricular and related nuclei of the hypothalamus; the periaqueductal gray matter; the nucleus of the solitary tract; the nucleus ambiguous; the ventrolateral medulla; the ventromedial medulla and the medullary tegmental field. The primary output of the CAN is mediated through the preganglionic sympathetic and parasympathetic neurons, which exert control over the heart via the stellate ganglia and the vagus nerve, respectively. The interplay of sympathetic and 
parasympathetic influences on sinoatrial node pacemaker activity generates the complex variability that characterizes the healthy heart rate rhythm, which is called HRV [9]. A fundamental principle of the neural control of the heart is its hierarchical organization, with cortical structures providing inhibitory control over limbic and brainstem sympathoexcitatory, cardioacceleratory circuits. Indeed, disruption of prefrontal activity leads to disinhibition of sympathoexcitatory circuits, with a resultant increase in heart rate and decrease in vagally-mediated HRV (37/Verberne, 1996; 38/Verberne, 1997). Furthermore, left-sided (dominant hemisphere) forebrain structures appear to be predominantly involved in vagal regulation, whereas homotopic right (nondominant) forebrain regions seem to primarily control sympathetic tone and responses (39/Guo et al., 2016; 40/Winkelmann T, 2017). However, the lateralization model of autonomic control of the heart remains controversial (41/Thayer et al., 2012; 42/Yoo, 2017; 43/Carnevali L, 2018).

We also found a positive correlation of Ap-indices with HRV sympathetic/vagal balance indices as well as a negative correlation with HRV-markers of vagal tone, but with a shift of a few days (see Table 10). It appears that geomagnetic perturbation-induced inhibition of vagal nuclei and/or excitation of sympathetic nuclei of the brainstem occurs only after a few days. On the other hand, inhibition of theta rhythm in loci C3, C4 and T6 on the day of registration of EEG and on the eve of it is preceded by its activation 3-4 days before registration. 5 days before registration, the delta rhythm in loci F8, Fp1 and T6 is activated as well as beta rhythm in locus $\mathrm{O} 2$, and 6 days before registration geomagnetic disturbance activates beta rhythm in loci F3 and C3, instead inhibits delta rhythm in locus F7.

The data obtained are consistent with those (obtained earlier by identical design in persons with a similar state of the body) about the significant correlation between the parameters of EEG and HRV [44/Popovych, 2013;45/Popovych,2014].

Novik and Smirnov (25/2013) studied the effect of geomagnetic storms at the latitude of Moscow on the electric oscillations of the human brain cerebral cortex. In course of electroencephalogram measurements it was shown that when the voluntary persons at the age of 18-23 years old were performing tasks using a computer during moderate magnetic storm or no later than $24 \mathrm{hrs}$ after it, the value of the coherence function of electric oscillations of the human brain in the frontal and occipital areas in a range of $4,0-7,9 \mathrm{~Hz}$ (the theta rhythm) decreased by a factor of two or more, sometimes reaching zero, although arterial blood pressure, respiratory rate and the electrocardiogram registered during electroencephalogram measurements remained within the standard values.

Baevsky et al [26/1997] discovered during 1990-1994 the about 30\% decrease in RMSSD of HRV in cosmonauts studied during a geomagnetic storm as compared to cosmonauts monitored on quiet days.

McCraty et al [27/2017] a coupling between geomagnetic activity and the human nervous system's function identified by virtue of continuous monitoring of HRV and the time-varying geomagnetic field over a 31-day period in a group of 10 individuals who went about their normal day-to-day lives. A time series correlation analysis identified a response of the group's autonomic nervous systems to various dynamic changes in the solar, cosmic ray, and ambient magnetic field. Correlation coefficients were calculated between the HRV variables and environmental measures during three distinct time periods of environmental activity. There were significant correlations between the group's HRV and solar wind speed, Kp, Ap, solar radio flux, cosmic ray counts, Schumann resonance power, and the total variations in the magnetic field. In addition, the time series data were time synchronized and normalized, after which all circadian rhythms were removed. It was found that the participants' HRV rhythms synchronized across the 31-day period at a period of approximately 2,5 days, even though all participants were in separate locations. Overall, this suggests that daily autonomic nervous system activity not only responds to changes in solar and geomagnetic activity, but is synchronized with the time-varying magnetic fields associated with geomagnetic field-line resonances and Schumann resonances.

If the disturbances of the Earth's magnetic field are an obvious causal factor, then we are tormented by doubts about its first target (acceptor). Accepting the hypothesis of Limansky [6,7] and the concept of nervousness, we conclude that caused by geomagnetic disturbances multidirectional and asymmetric changes in the conductivity of a limited constellation of APs in one way or another (through afferent cutaneous nerves or channels of structured liquid crystal water?) reach medullary, subcortical and cortical structures of CAN, exciting some neurons and inhibiting others, thus triggering well-studied mechanisms of neuro-immune, neuroendocrine and endocrine-immune modulation [16-19,44-51] documented in our study.

If we accept the paradigm of Eastern medicine, the information from APs enters the Chakras, which regulate the neuro-endocrine-immune complex. The reality of this assumption is evidenced by the parameters of GDV (biophotonics), registered in the same observed individuals. But this will be discussed in a separate article.

Mulligan et al [24/2010] suggest that direct geomagnetic effects on electroencephalographic activity can be reduced to the essential congruence of the magnitude of energy density within the human brain and the geomagnetic field. Their calculations showed that a shift of geomagnetic activity of only $25 \mathrm{nT}$ has the potential to change the energy available within the brain. Consequently, either the change in atmospheric power or the increase in geomagnetic intensity within this range would provide comparable changes in energy within the brain 
volume. Of course this is not a proof that energy absorption within the brain occurs from changes in either geomagnetic activity or atmospheric power.

According to Nordmann et al [52], there are 3 prevailing proposals being weighed regarding how magnetic fields are sensed by animals. First is provocation of action potentials in neurons by electromagnetic induction; second is light-sensitive, chemical-based mechanism mediated by cryptochromes resulting in action potentials as nervous signals [53-58]; and third is magnetite-based magnetoreceptors mechanically spotting the magnetic field, leading to neuronal action potentials [59-63].

Therefore, the brain can directly accept geomagnetic disturbances, without the mediation of APs, followed by neuro-endocrine and neuro-immune modulation. If this is accepted, then the changes in the electrical conductivity of APs detected by us should be considered as a consequence of the well-known skin-galvanic reflex caused by brain stimulation geomagnetic disturbances.

Simko and Mattsson [64/2004] envisage that electromagnetic field (EMF) exposure can cause both acute and chronic effects that are mediated by increased free radical levels. Firstly, direct activation of, for example macrophages (or other cells) by short-term exposure to EMF leads to phagocytosis (or other cell specific responses) and consequently, free radical production. This pathway may be utilized to positively influence certain aspects of the immune response. Secondly, EMF-induced macrophage (cell) activation includes direct stimulation of free radical production.

Rosado et al (65/2018) note, whereas the majority of the in vitro studies focused on monocytes/macrophages and fibroblasts, the effects of the exposure to EMF on other cell types are not well defined. In macrophages, the reduction in pro-inflammatory cytokines induced by ELF-EMF is associated with the activation of regulatory mechanisms induced by a moderate oxidative stress. However, in the case of neutrophils, activation of oxidative stress by ELF-EMF induces activation of NETosis.

However, this information is not entirely appropriate for our discussion, because the applied EMFs had a power comparable to that of the Earth's magnetic field or even greater. Only two studies of Selmaoui et al [66/1996;67/2011] is noteworthy. The authors showed that exposure to 50-Hz magnetic fields (10 $\mu$ T) significantly increases IL-6 when subjects were exposed to an intermittent magnetic field. However, no effect has been observed on interleukin IL-1 $\beta$, IL-2, IL-1RA, and IL-2R. No significant differences were observed between sham-exposed (control) and exposed men for hemoglobin concentration, hematocrit, red blood cells, platelets, total leukocytes, monocytes, lymphocytes, eosinophils, or neutrophils. Immunologic variables (CD3, CD4, CD8, NK and B cells) were unaltered.

Therefore, the initial acceptance of geomagnetic disturbances by immunocytes is possible, but unlikely.

We are pleased to consider Gorgo et al [68/2018] recent study. The monitoring of specific intensity of bacteria Photobacterium phosphoreum luminescence has been carried out for 2 months (September-October 2015) and it was compared to the daily values of activity of the geomagnetic field in the conditions of Kyïv, during the research of bioluminescence. Variation determination of the geomagnetic field was conducted from data of Space Environment Center, NOAA\&U.S. Air Force. The inverse proportional reliable average correlation was defined between the values of specific bacterial luminescence and the $\mathrm{Kp}(\mathrm{r}=-0,41)$ as well as Ap $(\mathrm{r}=-0,41)$ indexes of the geomagnetic field, and with the values of flux of Sun radiation at a wavelength 10,7 cm - reliable directly proportional correlation $(\mathrm{r}=0,305)$. Interestingly, the average values of Ap-indexes were close to those in our observations (in September 13,7 $\pm 2,68 \mathrm{nT}$; in October 14,9 $\pm 1,90 \mathrm{nT}$ ). Surprising is the proximity of the force of influence of the intensity of variations of the magnetic field on the day of testing (Ap0) both on the manifestation of bacterial activity and phagocytic function of neutrophils against bacteria. This, in our opinion, confirms the direct effect of the geomagnetic field disturbances on human neutrophils. The decrease in the level of T-helpers $(r=-0,27)$ and B-lymphocytes $(r=-0,19)$ reflects, in our opinion, the weakening of their expression of CD4 and CD22 receptors, respectively, as evidenced by the positive correlation of the Ap-index with the level of 0-lymphocytes $(r=0,26)$.

Assuming the hypothesis that immunocytes are the primary target of geomagnetic disturbances, we have reason to speculate that the detected changes in EEG and HRV parameters are caused by released immunocytes, primarily macrophages, cytokines, hormones and neurotransmitters [46,47].

Finally, the hypothesis that phagocytosis inhibition of two types of microbes found in our study may be due to the positive effects of geomagnetic disturbances directly on bacteria (for example bacteria Photobacterium phosphoreum), which are known to be able to actively counteract the bactericidal properties of phagocytes and other immunocytes, is not disqualified.

\section{ACKNOWLEDGMENT}

We express our sincere gratitude to administration of clinical sanatorium "Moldova" (Truskavets') for help in carrying out testing as well as Danylo Mel'nyk for Ap-index monitoring.

\section{ACCORDANCE TO ETHICS STANDARDS}


Tests in patients are conducted in accordance with positions of Helsinki Declaration 1975, revised and complemented in 2002, and directive of National Committee on ethics of scientific researches. During realization of tests from all participants the informed consent is got and used all measures for providing of anonymity of participants.

For all authors any conflict of interests is absent.

Р.Г. Церковнюк ${ }^{1}$, А.І. Гоженко ${ }^{1}$, Т.А. Королишин ${ }^{1,2,3}$, М.М. Ковбаснюк ${ }^{2}$ В.Й. Губицький ${ }^{1,3}$, В.В. Кіхтан $^{1,3}$, В.М. Філь ${ }^{4}$, А.С. Анчев ${ }^{1}$, В.А. Жуков ${ }^{4}$, Р.І. Янчій ${ }^{2}$, І.Л. Попович ${ }^{1,2}$

\title{
ВЗАСМОЗВ'ЯЗКИ МІЖ ГЕОМАГНІТНИМ Ар-ІНДЕКСОМ ТА ПАРАМЕТРАМИ ТОЧОК АКУПУНКТУРИ І НЕЙРОЕНДОКРИННО-ІМУННОГО КОМПЛЕКСУ У ПАЦІЕНТІВ 3 ЙОГО ДИСФУНКЦІЕЮ
}

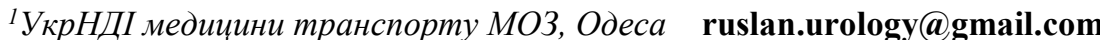 \\ ${ }^{2}$ Iнститут фізіологї ім. О.О. Богомольия НАH, Київ i.popovych@biph.kiev.ua \\ ${ }^{3}$ Клінічний санаторій “Молдова”, Трускавецьь \\ ${ }^{4}$ Nicolaus Copernicus University, Torun, Poland w.zukow@wp.pl
}

Передумова. Ще в 1990 році Ю. П. Лиманський висунув гіпотезу про акупунктурні точки як полімодальні рецептори системи екоцептивної чутливості. У процесі розвитку гіпотези у 2003 р. було обгрунтовано існування окремої функціональної системи регуляції електромагнітного балансу організму та сформульована робоча концепція світлолікування. В руслі даної гіпотези ми поставили перед собою мету проаналізувати зв'язки між збуреннями геомагнітного поля (за Ар-індексом) і електропровідністю низки точок акупунктури (ТА), з одного боку, і параметрами нейро-ендокринно-імунного комплексу у пацієнтів з його дисфункцією - 3 іншого боку. Матеріал і методи. Об'єктом спостереження були 21 чоловік (24-63р.) та 20 жінок (30-72 р.) 3 дисфункцією нейроендокринно-імунного комплексу. Кожного пацієнта обстежували двічі з інтервалом у 4 дні. Спостереження проводилися 09.06. та 13.06. 2015, 14.09 та 18.09. 2015, 27-28.03. та 04-05.04. 2018, 28.01. та 01.02. 2019. Ретроспективно реєстрували геомагнітний Ар-індекс в день тестування та протягом попередніх 7 днів, використовуючи ресурс https://www.spaceweatherlive.com/. Реєстрували електропровідність 9 пар ТА, параметри електроенцефалограми (ЕЕГ) і варіабельності ритму серця (ВРС), визначали концентрацію в плазмі кортизолу, трийодтироніну і тестостерону, вміст в крові субпопуляцій лімфоцитів, що експресують рецептори CD3, CD4, CD25, CD8, CD22 та CD56, а також сироваткову концентрацію циркулюючих імунних комплексів, імуноглобулінів класів M, G, A, C-реактивного білка та IL-1 $\beta$ i IL-6. Cтан фагоцитарної функції нейтрофілів оцінений за мікробним числом та індексами активності фагоцитозу і кілінгу Staphylococcus aureus i Escherichia coli. Результати. Протягом тижня середній рівень Ар-індексу коливався від 7 до 13 нТл. Максимальні коефіцієнти множинної кореляції Ар-індексу з параметрами ТА виявлені за 6 днів до $(\mathrm{R}=0,552)$ і в день їх реєстрації $(\mathrm{R}=0,470), 3$ параметрами ЕЕГ - напередодні реєстрації $(\mathrm{R}=0,708)$ та за 6 днів до неї $(\mathrm{R}=0,685)$, з параметрами імунітету - напередодні забору крові $(\mathrm{R}=0,768)$ і за 5 днів до нього $(\mathrm{R}=0,758)$, з показниками $\mathrm{BPC}$ і гормонів - за $2(\mathrm{R}=0,506)$ та $7(\mathrm{R}=0,403)$ днів до дослідження. Канонічна кореляція між Ар-індексами впродовж 7 днів до та в день тестування i параметрами ТА становить 0,661, ЕЕГ - 0,886, ВРС і гормонів - 0,766, параметрами імунітету - 0,921. Своєю чергою, параметри імунітету тісно пов'язані з параметрами ЕЕГ $(\mathrm{R}=0,944)$ та ВРС і гормонів $(\mathrm{R}=0,714)$. Висновок. Збурення геомагнітного поля (Ар-індекс) чинить значний негайний модулюючий вплив на параметри нейро-ендокринно-імунного комплексу, мабуть, через посередництво точок акупунктури як полімодальних рецепторів екоцептивної сенситивної системи.

Ключові слова: геомагнітний Ар-індекс, точки акупунктури, нейро-ендокринно-імунний комплекс, кореляції, люди.

\section{References}

1. Chizhevsky AL. The Terrestrial Echo of Solar Storms. Moscow. Mysl; 1976: 366. [in Russian].

2. Halberg F, Cornélissen G, Otsuka K, Watanabe Y, Katinas GS, Burioka N, Delyukov A, Gorgo Y et al. Cross-spectrally coherent $\sim 10.5$ - and 21-year biological and physical cycles, magnetic storms and myocardial infarctions. Neuro Endocrinology Letters. 2000; 21(3): 233-258.

3. Zhadin MN. Review of Russian literature on biological action of DC and low-frequency AC magnetic fields. Bioelectromagnetics. 2001; 22(1): 27-45.

4. Dubrov A. The Geomagnetic Field and Life: Geomagnetobiology. Springer; 2013.

5. Hanslmeier A. The Sun and Space Weather. 2. Dordrecht: Springer; 2007. 
6. Limansky YuP. Hypothesis about acupuncture points as polymodal receptors of the ecoceptive sensitivity system. Fiziol Zhurn. 1990; 36(4): 115-121. [in Russian].

7. Gulyar SA, Limansky YuP. Functional system of regulation of electromagnetic balance of organism: mechanisms of primary reception of electromagnetic waves of optical range. Fiziol Zhurn. 2003; 49(2): 35-44. [in Ukrainian].

8. Popovych IL, Gozhenko AI, Badiuk NS, Napierata M, Muszkieta R, Zukow W, Yanchij RI, Lapovets' NYe, Lapovets' LYe, Tserkovnyuk RG, Akimova VM, Nahurna YV, Martianova OI, Vivchar RYa, Chendey IV, Ruzhylo SV. Relationships between geomagnetic Ap-index and parameters of the immunity in patients with multiple sclerosis and radiculopathies. Journal of Education, Health and Sport. 2021; 11(3): 77-90.

9. Heart Rate Variability. Standards of Measurement, Physiological Interpretation, and Clinical Use. Task Force of ESC and NASPE. Circulation. 1996; 93(5): 1043-1065.

10. Berntson GG, Bigger JT jr, Eckberg DL, Grossman P, Kaufman PG, Malik M, Nagaraja HN, Porges SW, Saul JP, Stone PH, Van der Molen MW. Heart Rate Variability: Origines, methods, and interpretive caveats. Psychophysiology. 1997; 34: 623-648.

11. Baevskiy RM, Ivanov GG. Heart Rate Variability: theoretical aspects and possibilities of clinical application. Ultrazvukovaya i funktsionalnaya diagnostika. 2001; 3: 106-127. [in Russian].

12. Popadynets' OO, Gozhenko AI, Zukow W, Popovych IL. Peculiarities of spectral parameters of EEG, HRV and routine parameters of immunity in patients with various levels of the entropy of EEG, HRV, immunocytogram and leukocytogram. Journal of Education, Health and Sport. 2019; 9(8): 617-636.

13. Popadynets' O, Gozhenko A, Badyuk N, Popovych I, Skaliy A, Hagner-Derengowska M et al. Interpersonal differences caused by adaptogen changes in entropies of EEG, HRV, immunocytogram, and leukocytogram. Journal of Physical Education and Sport. 2020; 20(Suppl. 2): 982-999.

14. Lapovets' LYe, Lutsyk BD. Laboratory Immunology [in Ukrainian]. Kyiv; 2004: 173.

15. Douglas SD, Quie PG. Investigation of Phagocytes in Disease. Churchil; 1981: 110.

16. Kul'chyns'kyi AB, Kovbasnyuk MM, Kyjenko VM., Zukow W, Popovych IL. Neuro-immune relationships at patients with chronic pyelonephrite and cholecystite. Communication 2. Correlations between parameters EEG, HRV and Phagocytosis. Journal of Education, Health and Sport. 2016; 6(10): 377-401.

17. Popovych IL, Kul'chyns'kyi AB, Gozhenko AI, Zukow W, Kovbasnyuk MM, Korolyshyn TA. Interrelations between changes in parameters of HRV, EEG and phagocytosis at patients with chronic pyelonephritis and cholecystitis. Journal of Education, Health and Sport. 2018; 8(2): 135-156.

18. Gozhenko AI, Zukow W, Polovynko IS, Zajats LM, Yanchij RI, Portnichenko VI, Popovych IL. Individual Immune Responses to Chronic Stress and their Neuro-Endocrine Accompaniment. RSW. UMK. Radom. Torun; 2019: 200.

19. Gozhenko AI, Korda MM, Popadynets' OO, Popovych IL. Entropy, Harmony, Synchronization and their Neuro-endocrine-immune Correlates. Odesa. Feniks; 2021: 232. [in Ukrainian].

20. Popovych IL. Information effects of bioactive water Naftyssya in rats: modulation entropic, prevention desynchronizing and limitation of disharmonizing actions water immersion stress for information components of neuro-endocrine-immune system and metabolism, which correlates with gastroprotective effect. Medical Hydrology and Rehabilitation. 2007; 5(3): 50-70. [in Ukrainian].

21. Tserkovniuk R, Yanchij R, Plyska O, Kovbasnyuk M, Chendey I, Hagner-Derengowska M, Zukow X, Kałuzny K, Muskieta R, Zukow W. Relationships between geomagnetic Ap-index and parameters of the immunity in patients with neuroendocrine-immune complex dysfunction. PharmacologyOnLine. 2021; 3:

22. Muehsam D, Ventura C. Life rhythm as a symphony of oscillatory patterns: electromagnetic energy and sound vibration modulates gene expression for biological signaling and healing. Glob Adv Health Med. 2014; 3(2): 40-55. doi:10.7453/gahmj.2014.008.

23. Babayev ES, Allahverdiyeva AA. Effects of geomagnetic activity variations on the physiological and psychological state of functionally healthy humans: some of results of the Azerbijani studies. Advances in Space Research. 2007; 40: 1941-1951.

24. Mulligan BP, Hunter MD, Persinger MA. Effects of geomagnetic activity and atmospheric power variations on quantitative measures of brain activity: replication of the Azerbaijani studies. Advances in Space Research. 2010; 45: 940-948.

25. Novik OB, Smirnov FA. Geomagnetic storm decreases coherence of electric oscillations of human brain while working at the computer. Biofizika. 2013;58(3):554-560.

26. Baevsky RM, Petrov VM, Cornelissen G, Halberg F, Orth-Gomer K, Akerstedt T, Otsuka K, Breus T, Siegelova J, Dusek J, Fiser B. Meta-analyzed heart rate variability, exposure to geomagnetic storms, and the risk of ischemic heart disease. Scr Med (Brno). 1997; 70(4-5): 201-206. 
27. McCraty, R., Atkinson, M., Stolc, V., Alabdulgader, A. A., Vainoras, A., \& Ragulskis, M. (2017). Synchronization of Human Autonomic Nervous System Rhythms with Geomagnetic Activity in Human Subjects. International journal of environmental research and public health, 14(7), 770. https://doi.org/10.3390/ijerph14070770

28. Persinger, M.A., Richards, P.M. Vestibular experiences of humans during brief periods of partial sensory deprivation are enhanced when daily geomagnetic activity exceeds 15-20 nT. Neuroscience Letters 194, 69-72, 1995

29. Cherry, N. Schumann resonance, a plausible biophysical mechanism for the human health effects of solar/geomagnetic activity. Natural Hazards 26, 279-331, 2002.

30. Schlegel, K., Fuellekrug, K. Schumann resonance parameter changesduring high-energy particle precipitation. Journal of Geophysical Research 104, 10,111-10,118, 1999

31. Koenig, H.L., Krueger, A.P., Lang, S., Sonning, W. Biologic Effects of Environmental Electromagnetism. Springer-Verlag, New York, 1981.

32. Buzsaki, G. Theta oscillations in the hippocampus. Neuron 33, 325-340,2002.

33. Gloor, P. The Temporal Lobe and Limbic System. Oxford, New York,1997.

34. Benarroch EE. The central autonomic network: functional organization, dysfunction, and perspective. Mayo Clin Proc. 1993;68(10):988-1001. doi:10.1016/s0025-6196(12)62272-1

35. Palma, J. A., and Benarroch, E. E. (2014). Neural control of the heart: recent concepts and clinical correlations. Neurology 83, 261-271.doi: 10.1212/WNL.0000000000000605

36. Thayer JF, Lane RD. Claude Bernard and the heart-brain connection: further elaboration of a model of neurovisceral integration. Neurosci Biobehav Rev. 2009; 33(2): 81-88. doi:10.1016/j.neubiorev.2008.08.004

37. Verberne AJ. Medullary sympathoexcitatory neurons are inhibited by activation of the medial prefrontal cortex in the rat. Am J Physiol. 1996; 270 (4Pt2): R713-R719. doi:10.1152/ajpregu.1996.270.4.R713

38. Verberne AJ, Lam W, Owens NC, Sartor D. Supramedullary modulation of sympathetic vasomotor function. Clin Exp Pharmacol Physiol. 1997;24(9-10):748-754. doi:10.1111/j.14401681.1997.tb02126.x

39. Guo, C. C., Sturm, V. E., Zhou, J., Gennatas, E. D., Trujillo, A. J., Hua, A. Y.,et al. (2016). Dominant hemisphere lateralization of cortical parasympathetic control as revealed by frontotemporal dementia. Proc. Natl. Acad. Sci. U.S.A.113, E2430-E2439. doi: 10.1073/pnas.1509184113

40. Winkelmann T, Thayer JF, Pohlack S, Nees F, Grimm O, Flor H. Structural brain correlates of heart rate variability in a healthy young adult population. Brain Struct Funct. 2017;222(2):1061-1068. doi:10.1007/s00429-016-1185-1

41. Thayer, J. F., Ahs, F., Fredrikson, M., Sollers, J. J. III, and Wager, T. D. (2012). A meta-analysis of heart rate variability and neuroimaging studies: implications for heart rate variability as a marker of stress and health. Neurosci Biobehav Rev. 36, 747-756. doi: 10.1016/j.neubiorev.2011.11.009

42. Yoo HJ, Thayer JF, Greening S, et al. Brain structural concomitants of resting state heart rate variability in the young and old: evidence from two independent samples. Brain Struct Funct. 2018;223(2):727737. doi:10.1007/s00429-017-1519-7

43. Carnevali L, Koenig J, Sgoifo A, Ottaviani C. Autonomic and Brain Morphological Predictors of Stress Resilience Front Neurosci. 2018; 12: 228.

44. Popovych IL, Lukovych YuS, Korolyshyn TA, Barylyak LG, Kovalska LB, Zukow W. Relationship between the parameters heart rate variability and background EEG activity in healthy men. Journal of Health Sciences. 2013; 3(4): 217-240.

45. Popovych IL, Kozyavkina OV, Kozyavkina NV, Korolyshyn TA, Lukovych YuS, Barylyak LG. Correlation between Indices of the Heart Rate Variability and Parameters of Ongoing EEG in Patients Suffering from Chronic Renal Pathology. Neurophysiology. 2014; 46(2): 139-148.

46. Tracey KJ. Physiology and immunology of the cholinergic antiinflammatory pathway. J Clin Invest. 2007; 117(2): 289-296.

47. Thayer JF, Sternberg EM. Neural aspects of immunomodulation: Focus on the vagus nerve. Brain Behav Immun. 2010; 24(8): 1223-1228.

48. Kul'chyns'kyi AB, Gozhenko AI, Zukow W, Popovych IL. Neuro-immune relationships at patients with chronic pyelonephrite and cholecystite. Communication 3. Correlations between parameters EEG, HRV and Immunogram. Journal of Education, Health and Sport. 2017; 7(3): 53-71.

49. Kul'chyns'kyi AB, Kyjenko VM, Zukow W, Popovych IL. Causal neuro-immune relationships at patients with chronic pyelonephritis and cholecystitis. Correlations between parameters EEG, HRV and white blood cell count. Open Medicine. 2017; 12(1): 201-213.

50. Kul'chyns'kyi AB, Zukow W, Korolyshyn TA, Popovych IL. Interrelations between changes in parameters of HRV, EEG and humoral immunity at patients with chronic pyelonephritis and cholecystitis. Journal of Education, Health and Sport. 2017; 7(9): 439-459. 
51. Popovych IL, Kul'chyns'kyi AB, Korolyshyn TA, Zukow W. Interrelations between changes in parameters of HRV, EEG and cellular immunity at patients with chronic pyelonephritis and cholecystitis. Journal of Education, Health and Sport. 2017; 7(10): 11-23.

52. Nordmann G.C., Hochstoeger T., Keays D.A. Unsolved mysteries: magnetoreception - A sense without a receptor. PLoS Biol. 2017;15(10): e2003234.

53. Gegear, R. J., Casselman, A., Waddell, S., Reppert, S. M. (2008). Cryptochrome mediates lightdependent magnetosensitivity in Drosophila. Nature, 454(7207), 1014-1018. https://doi.org/10.1038/nature07183

54. Foley, L., Gegear, R., Reppert, S. Human cryptochrome exhibits light-dependent magnetosensitivity. Nat Commun 2, 356 (2011). https://doi.org/10.1038/ncomms1364

55. Zaporozhan V, Ponomarenko A. Mechanisms of geomagnetic field influence on gene expression using influenza as a model system: basics of physical epidemiology. Int J Environ Res Public Health. 2010; 7(3): 938-965. doi:10.3390/ijerph7030938.

56. Hammad, M., Albaqami, M., Pooam, M., Kernevez, E., Witczak, J., Ritz, T., Martino, C., \& Ahmad, M. (2020). Cryptochrome mediated magnetic sensitivity in Arabidopsis occurs independently of lightinduced electron transfer to the flavin. Photochemical \& photobiological sciences, 19(3), 341-352. https://doi.org/10.1039/c9pp00469f

57. Cifra, M., Apollonio, F., Liberti, M., García-Sánchez, T., Mir, L. M. (2021). Possible molecular and cellular mechanisms at the basis of atmospheric electromagnetic field bioeffects. International journal of biometeorology, 65(1), 59-67. https://doi.org/10.1007/s00484-020-01885-1

58. Wan, G., Hayden, A. N., Iiams, S. E., \& Merlin, C. (2021). Cryptochrome 1 mediates light-dependent inclination magnetosensing in monarch butterflies. Nature communications, 12(1), 771. https://doi.org/10.1038/s41467-021-21002-z

59. Kirschvink JL, Kobayashi-Kirschvink A, Woodford BJ. Magnetite biomineralization in the human brain. Proc Natl Acad Sci USA. 1992; 89(16): 7683-7687. doi: 10.1073 /pnas.89. 16. 7683.

60. Kirschvink JL, Kobayashi-Kirschvink A, Diaz-Ricci JC, Kirschvink SJ. Magnetite in human tissues: a mechanism for the biological effects of weak ELF magnetic fields. Bioelectromagnetics. 1992; Suppl 1: 101-113.

61. Kirschvink J.L., Walker M.M., Diebel C.E. Magnetite-based magnetoreception. Curr. Opin. Neurobiol. 2001;11(4):462-467.

62. Winklhofer M., Kirschvink J.L. A quantitative assessment of torque-transducer models for magnetoreception. J.R. Soc. Interface. 2010;7(suppl_2): S273-S289.

63. Gilder S.A., Wack M., Kaub L., Roud S.C., Petersen N., Heinsen H. et al. Distribution of magnetic remanence carriers in the human brain. Sci. Rep. 2018;8(1):1-9.

64. Simko M, Mattsson MO. Extremely low frequency electromagnetic fields as effectors of cellular responses in vitro: possible immune cell activation. J Cell Biochem. 2004; 93(1): 83-92. doi: 10.1002/jcb.20198.

65. Rosado, M. M., Simkó, M., Mattsson, M. O., \& Pioli, C. (2018). Immune-Modulating Perspectives for Low Frequency Electromagnetic Fields in Innate Immunity. Frontiers in public health, $6,85$. https://doi.org/10.3389/fpubh.2018.00085

66. Selmaoui B, Bogdan A, Auzeby A, Lambrozo J, Touitou Y. Acute exposure to $50 \mathrm{~Hz}$ magnetic field does not affect hematologic or immunologic functions in healthy young men: a circadian study. Bioelectromagnetics. 1996;17(5):364-372.

67. Selmaoui B, Lambrozo J, Sackett-Lundeen L, Haus E, Touitou Y. Acute exposure to 50-Hz magnetic fields increases interleukin-6 in young healthy men. J Clin Immunol (2011) 31(6):1105-11. doi:10.1007/s10875-011-9558-y

68. Gorgo YuP, Greckiy IO, Demydova OI. The use of luminos bacteria Photobacterium phosphoreum as a bioindicator of geomagnetic activity. Innov Biosyst Bioeng. 2018; 2(4): 271-277. doi:10.20535/ibb.2018.2.4.151459. 